\title{
Testing for Unit Roots Under Multiple Possible Trend Breaks and Non-Stationary Volatility Using Bootstrap Minimum Dickey-Fuller Statistics*
}

\author{
Giuseppe Cavaliere $^{a}$, David I. Harvey ${ }^{b}$, Stephen J. Leybourne ${ }^{b}$ \\ and A.M. Robert Taylor ${ }^{c}$ \\ ${ }^{a}$ Department of Statistical Sciences, University of Bologna \\ ${ }^{b}$ School of Economics, University of Nottingham \\ ${ }^{c}$ Essex Business School, University of Essex
}

January 2014

\begin{abstract}
In a recent paper, Harvey et al. (2013) [HLT] propose a new unit root test that allows for the possibility of multiple breaks in trend. Their proposed test is based on the infimum of the sequence (across all candidate break points) of local GLS detrended augmented Dickey-Fuller-type statistics. HLT show that the power of their unit root test is robust to the magnitude of any trend breaks. In contrast, HLT show that the power of the only alternative available procedure of Carrion-i-Silvestre et al. (2009), which employs a pre-test-based approach, can be very low indeed (even zero) for the magnitudes of trend breaks typically observed in practice. Both HLT and Carrion-i-Silvestre et al. (2009) base their approaches on the assumption of homoskedastic shocks. In this paper we analyse the impact of non-stationary volatility (for example single and multiple abrupt variance breaks, smooth transition variance breaks, and trending variances) on the tests proposed in HLT. We show that the limiting null distribution of the HLT unit root test statistic is not pivotal under nonstationary volatility. A solution to the problem, which does not require the practitioner to specify a parametric model for volatility, is provided using the wild bootstrap and is shown to perform well in practice. A number of different possible implementations of the bootstrap algorithm are discussed.
\end{abstract}

Keywords: Infimum unit root test; multiple trend break; non-stationary volatility; wild bootstrap. JEL Classification: C22.

\footnotetext{
${ }^{*}$ Financial support provided by the Economic and Social Research Council of the United Kingdom under research grant RES-000-22-3882 is gratefully acknowledged by the authors. Correspondence to: Robert Taylor, Essex Business School, University of Essex, Colchester, CO4 3SQ, UK. E-mail: rtaylor@essex.ac.uk
} 


\section{Introduction}

Given the apparent prevalence of deterministic breaks in trend observed in macroeconomic time series data, it is now common practice to allow for such structural change when conducting unit root tests. Initial work by Perron (1989) assumed the location of a potential single trend break to be known, but more recent approaches have focused on the case where the possible break occurs at an unknown point in the sample; see, inter alia, Zivot and Andrews (1992) [ZA], Banerjee et al. (1992), Perron (1997) and Perron and Rodríguez (2003) [PR]. An important issue surrounding such procedures is that there is also an underlying problem of uncertainty as to whether trend breaks exist in the data or not. To illustrate the point, when a single trend break is known to be present, the test based on PR's local GLS detrended ADF statistic which allows for a trend break is (near) asymptotically efficient. This holds provided the break point is known, or can be dated endogenously with sufficient precision. However, when a trend break does not occur the PR test is not asymptotically efficient, the redundant trend break regressor compromising power. Moreover, the asymptotic critical values for the PR test based on an estimated break point differ markedly according to whether a trend break occurs or not.

In response to this problem, Kim and Perron (2009), Carrion-i-Silvestre et al. (2009) [CKP] and Harris et al. (2009) [HHLT] focused on developing testing procedures which utilize auxiliary statistics to detect the presence of trend break(s) occurring at unknown point(s) in the sample, and then use the outcome of the detection step to indicate whether or not the unit root test employed should include trend break(s) in the deterministic specification. Assuming the trend break magnitudes to be fixed (independent of sample size), CKP and HHLT show their methods achieve asymptotically efficient unit root inference in both the no trend break and trend break environments. Crucially they assume the trend break magnitude(s) to be fixed, which renders the trend break pre-tests used in these procedures consistent against breaks of fixed magnitude and so the correct unit root test variant (either allowing for trend breaks or not) is applied in large samples. However, in finite samples the pre-tests will not provide perfect discrimination; i.e., some degree of uncertainty will necessarily exist in finite samples as to whether breaks are present or not. As a result, the asymptotic properties of these procedures contrast sharply with the finite sample simulations reported in CKP and HHLT which show the presence of pronounced "valleys" in the finite sample power functions (mapped as functions of the break magnitudes), such that power is initially high for very small breaks, then decreases as the break magnitudes increase, before increasing again.

Harvey et al. (2012) show that treating the trend break magnitudes to be local-to-zero (in a Pitman drift sense), rather than fixed, allows the (local) asymptotic distribution theory to very closely approximate this finite sample power valley phenomenon. This is because the local-to-zero model for the breaks reflects in the asymptotic theory the uncertainty that necessarily exists in finite samples as to whether trend breaks are present in the data or not. Harvey et al. (2013) [HLT] show that the valleys problem worsens as the number of trend breaks present increases, other things being equal. HLT argue that the typical trend break magnitudes seen with real macroeconomic data lie well within these valley regions, suggesting that the CKP and HHLT methods may then be very poor at 
discriminating between the unit root null and stochastic stationary alternative in practice.

In response to these issues, HLT advocate an approach along the lines of that outlined by ZA and $\mathrm{PR}$ for the case of a single putative trend break. ZA and PR propose using the infimum of $t$-ratio-type OLS and local GLS detrended ADF statistics, respectively, taken across all candidate break points in a trimmed range. HLT establish the result that, unlike the ZA test which can have an asymptotic size which approaches one when a trend break occurs under the unit root null (see Vogelsang and Perron, 1998), the asymptotic size of the local GLS de-trended variant of PR, when run using asymptotic critical values relevant to the no break case, does not exceed the nominal level where either a fixed or a local-to-zero trend break occurs under the null. HLT generalise the contribution of PR by developing a local GLS detrended infimum test which allows for multiple possible breaks in trend, again based on asymptotic critical values which assume no breaks are present. They show that the local GLS detrended infimum test eliminates the aforementioned power valleys. This necessarily comes at the expense of some loss of power relative to the CKP test when no breaks are present. In a local-to-zero trend break environment and where the putative break fractions are unknown it is not possible to obtain unit root tests which are invariant (even asymptotically) to the break magnitudes, since the unknown break fractions cannot be consistently estimated. HLT argue that the infimum tests they propose come as close as one can come to achieving invariant inference under local trend breaks.

While the unit root test proposed in HLT allows for the possibility of breaks in the deterministic trend function, importantly HLT make no allowance for time-varying behaviour in the unconditional volatility of the driving shocks. In this paper we analyse the impact of non-stationary volatility in the shocks on the infimum test of HLT, and demonstrate that the asymptotic distribution of the HLT statistic is not pivotal and depends on the structure of the underlying volatility process. Simulation results suggest that this can have a large impact on both the size (and power) properties of the infimum test, most critically leading to severe over-size in certain cases. Since, for many macroeconomic series, non-constancy in the unconditional volatility of the shocks appears to be a relatively common phenomenon (see Cavaliere and Taylor, 2008, and the references therein), we consider approaches that attempt to overcome this inference problem. Specifially, we propose an implementation of the HLT test using the wild bootstrap principle, replicating in the re-sampled data the essential pattern of heteroskedasticity present in the original shocks (which might include, for example, single or multiple abrupt variance breaks, smooth transition variance breaks, or trending variances). The wild bootstrap approach has proven to be effective in the case of standard unit root tests which allow for either a constant or linear trend; see Cavaliere and Taylor (2008); moreover, Cavaliere et al. (2011) show that it can also be successfully applied to the single putative trend break unit root test of HHLT, although the power "valley" problem associated with HHLT still remains.

We consider a number of possible wild bootstrap-based procedures, none of which require the user to specify any parametric model of volatility. The leading bootstrap test we consider is based on re-sampling from the double differences of the original data. Double differencing is used since this transforms any trend breaks present into outliers which have no impact in large samples. We demonstrate that the resulting bootstrap infimum statistic shares the same limiting null distribution, when 
evaluated under the case of no breaks in trend, as the original infimum statistic of HLT under the class of non-stationary volatility considered. This ensures that in the no-break case, the bootstrap procedure is asymptotically correctly sized and also incurs no loss of asymptotic local power relative to the original HLT test. In the more general setting of non-zero local breaks in trend, we demonstrate that, in contrast to the HLT test, asymptotic over-size is now almost entirely absent. Other bootstrap algorithms discussed include those which explicitly model the trend break component (using an estimate of the break dates and break magnitudes) and, optionally, re-build the resulting estimated trend break component back into the bootstrap data. Re-building the estimated trend break component into the bootstrap data implies that, where trend breaks occur and are consistently estimated (the latter requires the break magnitudes to be fixed and non-zero), the bootstrap statistic will replicate the true asymptotic null distribution of the infimum statistic, rather than the limiting distribution of that statistic appropriate for the no break case. This might be expected to improve power in the case where trend breaks are present in the data given the observation of HLT that their test based on no-break critical values has a tendency to be under-sized where breaks occur. However, for zero and local magnitude trend breaks such a bootstrap statistic will not replicate the correct limiting null distribution and so will not be correctly sized. The finite sample size and power performance of these various bootstrap procedures are compared using Monte Carlo methods.

The paper is organised as follows. Our reference heteroskedastic multiple trend break model is outlined in section 2. Section 3 reviews the contribution of HLT. Section 4 details the large sample behaviour of the HLT unit root test statistic when the errors display non-stationary volatility. In section 5 we outline our leading wild bootstrap-based implementations of the HLT test and establish its asymptotic properties; we also describe the alternative bootstrap algorithms that we consider. Simulation evidence presented in Section 6 suggests that the proposed bootstrap tests perform well in small samples. Section 7 concludes. Proofs are collected in an Appendix.

In what follows we use the following notation: $\lfloor\cdot\rfloor$ denotes the integer part; o denotes the Hadamard product (i.e. element-wise multiplication); $\stackrel{w}{\rightarrow}$ denotes weak convergence, $\stackrel{p}{\rightarrow}$ convergence in probability, and $\stackrel{w}{\rightarrow} p$ weak convergence in probability (see, for example, Giné and Zinn, 1990), in each case as the sample size diverges; $1($.$) denotes the indicator function, and \mathbb{I}_{x}:=1(x \neq 0)$ and $\mathbb{I}_{x}^{y}:=1(y>x) ; x:=y$ $(x=: y)$ indicates that $x$ is defined by $y$ ( $y$ is defined by $x$ ), and $\stackrel{a}{=}$ denotes asymptotic equivalence; finally, $\mathcal{C}:=C[0,1]$ denotes the space of continuous processes on $[0,1]$, and $\mathcal{D}:=D[0,1]$ the space of right continuous with left limit (càdlàg) processes on $[0,1]$. 


\section{The Heteroskedastic Multiple Trend Break Model}

We consider the time series process $\left\{y_{t}\right\}$ generated according to the following model,

$$
\begin{aligned}
y_{t} & =\mu+\beta t+\gamma^{\prime} \mathbf{D} \mathbf{T}_{t}\left(\boldsymbol{\tau}_{0}\right)+u_{t}, \quad t=1, \ldots, T, \\
u_{t} & =\rho_{T} u_{t-1}+\varepsilon_{t}, \quad t=2, \ldots, T, \\
\varepsilon_{t} & =C(L) e_{t}=\sum_{j=0}^{\infty} c_{j} e_{t-j}, \\
e_{t} & =\sigma_{t} z_{t}
\end{aligned}
$$

where $\mathbf{D T}_{t}\left(\boldsymbol{\tau}_{0}\right):=\left[D T_{t}\left(\tau_{0,1}\right), \ldots, D T_{t}\left(\tau_{0, m}\right)\right]^{\prime}$, the elements of which, for a generic fraction $\tau$, are the indicator variables, $D T_{t}(\tau):=1(t>\lfloor\tau T\rfloor)(t-\lfloor\tau T\rfloor)$. In this model $\boldsymbol{\tau}_{0}:=\left[\tau_{0,1}, \ldots, \tau_{0, m}\right]^{\prime}$ is the vector of (unknown) putative trend break fractions, with $\gamma:=\left(\gamma_{1}, \ldots, \gamma_{m}\right)^{\prime}$ the associated break magnitude parameters; a trend break therefore occurs in $\left\{y_{t}\right\}$ at time $\left\lfloor\tau_{0, i} T\right\rfloor$ when $\gamma_{i} \neq 0, i=1, \ldots, m$. The break fractions are assumed to be such that $\tau_{0, i} \in \Lambda$, for all $i$, where $\Lambda:=\left[\tau_{L}, \tau_{U}\right]$ with $0<\tau_{L}<\tau_{U}<1$; the fractions $\tau_{L}$ and $\tau_{U}$ representing trimming parameters. It is also assumed that $\left|\tau_{0, i}-\tau_{0, j}\right| \geq \eta>0$, for all $i, j, i \neq j$, such that the DGP admits (up to) $m$ level breaks occurring at unknown points across the interval $\Lambda$, with a sample fraction of at least $\lfloor\eta T\rfloor$ observations between breaks. Notice, therefore, that $m$ and $\eta$ must satisfy the relation $m \leq 1+\left\lfloor\left(\tau_{U}-\tau_{L}\right) / \eta\right\rfloor^{1}$

In (2.2), $\left\{u_{t}\right\}$ is an unobserved mean zero stochastic process, initialised such that $u_{1}=o_{p}\left(T^{1 / 2}\right)$. The following set of assumptions will also be taken to hold on (2.1)-(2.4).

Assumption $\mathcal{A}: \mathcal{A}_{1}$. The lag polynomial satisfies $C(z) \neq 0$ for all $|z| \leq 1$, and $\sum_{j=0}^{\infty} j\left|c_{j}\right|<\infty ; \mathcal{A}_{2}$. $z_{t} \sim \operatorname{IID}(0,1)$ with $E\left|z_{t}\right|^{r}<K<\infty$ for some $r \geq 4 ; \mathcal{A}_{3}$. The volatility term $\sigma_{t}$ satisfies $\sigma_{t}=\omega(t / T)$, where $\omega(\cdot) \in \mathcal{D}$ is non-stochastic and strictly positive. For $t \leq 0, \sigma_{t} \leq \breve{\sigma}<\infty$.

Remark 1. Notice that $\left\{\varepsilon_{t}\right\}$ in (2.3) is a linear process in $\left\{e_{t}\right\}$, the latter formed as the product of two components, $\left\{z_{t}\right\}$ and $\left\{\sigma_{t}\right\}$. Since, under Assumption $\mathcal{A}_{2},\left\{z_{t}\right\}$ is IID, conditionally on $\sigma_{t}$, the error term $e_{t}$ has mean zero and time-varying variance $\sigma_{t}^{2}$.

Remark 2. Before progressing it is worth commenting that, since the variance $\sigma_{t}^{2}$ depends on $T$, a time series generated according to (2.1)-(2.4) with $\sigma_{t}$ satisfying Assumption $\mathcal{A}_{3}$ formally constitutes a triangular array of the type $\left\{y_{T, t}:=d_{T, t}+u_{T, t}: 1 \leq t \leq T, T \geq 2\right\}$, where $d_{T, t}$ is purely deterministic and $u_{T, t}$ is recursively defined as $u_{T, t}:=\rho_{T} u_{T, t-1}+C(L) \sigma_{T, t} z_{t}, \sigma_{T,\lfloor s T\rfloor}:=\omega(s)$. However, since the triangular array notation is not essential, for simplicity the subscript $T$ will be suppressed in what follows.

Assumption $\mathcal{A}$ coincides with the set of conditions adopted in Cavaliere and Taylor (2008). Assumption $\mathcal{A}_{1}$ is standard in the unit root literature. Assumption $\mathcal{A}_{2}$ is somewhat stronger than is often seen, since it rules out certain forms of conditional heteroskedasticity, such as that arising from

\footnotetext{
${ }^{1}$ One might also consider a second model which allows for simultaneous breaks in the level of the process. However, as noted by PR, pp.2,4, a change in intercept is a special case of a slowly evolving deterministic component (see Condition B of Elliott et al.,1996, p.816) and, consequently, does not alter any of the large sample results presented in this paper.
} 
stationary GARCH models, in the errors. It is made to simplify exposition; the results stated in this paper would continue to hold if this assumption was weakened along the lines detailed in Remark 1 of Cavaliere and Taylor (2008,pp.46-47). The key assumption for the purposes of this paper is $\mathcal{A}_{3}$, which only requires of the innovation variance that it is non-stochastic, bounded and displays a countable number of jumps. A detailed discussion of the class of variance processes allowed under $\mathcal{A}_{3}$ is given in Cavaliere and Taylor (2007); this includes variance processes displaying (possibly) multiple one-time volatility shifts (which need not be located at the same point in the sample as the putative trend breaks), polynomially (possibly piecewise) trending volatility and smooth transition variance breaks, among other things. The conventional homoskedasticity assumption, as employed in HLT, that $\sigma_{t}=\sigma$ for all $t$, also satisfies Assumption $\mathcal{A}_{3}$, since here $\omega(s)=\sigma$ for all $s$. Although Assumption $\mathcal{A}_{3}$ imposes the volatility process to be non-stochastic, this may be weakened along the same lines as are detailed in Remark 2 of Cavaliere and Taylor (2008,p.47).

A quantity which will play a key role in what follows is given by the following function in $\mathcal{C}$, known as the variance profile of the process:

$$
\eta(s):=\left(\int_{0}^{1} \omega(r)^{2} d r\right)^{-1} \int_{0}^{s} \omega(r)^{2} d r .
$$

Observe that the variance profile satisfies $\eta(s)=s$ under homoskedasticity while it deviates from $s$ in the presence of heteroskedasticity. Notice also that the quantity $\bar{\omega}^{2}:=\int_{0}^{1} \omega(r)^{2} d r$ which appears in (2.5), by Assumption $\mathcal{A}_{3}$ equals the limit of $T^{-1} \sum_{t=1}^{T} \sigma_{t}^{2}$, and may therefore be interpreted as the (asymptotic) average innovation variance. We will also use the result

$$
T^{-1 / 2} \sum_{t=1}^{\lfloor r T\rfloor} \sigma_{t} z_{t} \stackrel{w}{\rightarrow} \bar{\omega} W_{0}^{\eta}(r)
$$

where the process $W_{0}^{\eta}(r):=\int_{0}^{r} d W(\eta(s))$ is known as a variance-transformed Brownian motion, i.e. a Brownian motion under a modification of the time domain; see, for example, Davidson (1994).

\section{$3 \quad$ HLT's Infimum Test}

Our interest centres on testing the unit root null hypothesis $H_{0}: \rho_{T}=1$, against the local alternative, $H_{c}: \rho_{T}=1-c / T, c>0$. Under the assumption of homoskedastic innovations, that is $\sigma_{t}=\sigma$ for all $t$, HLT develop a test of $H_{0}$ against $H_{c}$ which does not require the practitioner to assume knowledge of whether trend breaks are present in the data or not. The test they propose is a multiple break version of the minimum local GLS detrended Dickey-Fuller statistic proposed by PR (which is $M D F_{1}$ in the notation below), following the approach taken by ZA in a single break OLS detrending environment. Specifically, the test statistic proposed in HLT is

$$
M D F_{m}:=\inf _{\substack{\tau_{1}, \ldots, \tau_{m} \in \Lambda,\left|\tau_{i}-\tau_{j}\right| \geq \eta, \forall_{i \neq j}}} D F_{\bar{c}}^{G L S}(\boldsymbol{\tau})
$$


where $D F_{\bar{c}}^{G L S}(\boldsymbol{\tau})$ denotes the standard $t$-ratio associated with $\hat{\pi}$ in the fitted OLS ADF-type regression

$$
\Delta \tilde{u}_{t}=\hat{\pi} \tilde{u}_{t-1}+\sum_{j=1}^{p} \hat{\psi}_{j} \Delta \tilde{u}_{t-j}+\hat{e}_{t}, \quad t=p+2, \ldots, T,
$$

where $\tilde{u}_{t}:=y_{t}-\tilde{\mu}-\tilde{\beta} t-\tilde{\gamma}^{\prime} \mathbf{D} \mathbf{T}_{t}(\boldsymbol{\tau})$, with $\left[\tilde{\mu}, \tilde{\beta}, \tilde{\gamma}^{\prime}\right]^{\prime}$ obtained from a local GLS regression of $\mathbf{y}_{\bar{\rho}}:=$ $\left[y_{1}, y_{2}-\bar{\rho} y_{1}, \ldots, y_{T}-\bar{\rho} y_{T-1}\right]^{\prime}$ on $\mathbf{Z}_{\bar{\rho}, \tau}:=\left[\mathbf{z}_{1}, \mathbf{z}_{2}-\bar{\rho} \mathbf{z}_{1}, \ldots, \mathbf{z}_{T}-\bar{\rho} \mathbf{z}_{T-1}\right]^{\prime}, \mathbf{z}_{t}:=\left[1, t, \mathbf{D} \mathbf{T}_{t}(\boldsymbol{\tau})^{\prime}\right]^{\prime}$ with $\bar{\rho}:=1-\bar{c} / T$, for some $\bar{c}>0$ which is user-supplied. ${ }^{2}$ This infimum unit root test rejects for large negative values of the statistic; HLT provide recommended values of $\bar{c}$, as well as asymptotic critical values relevant to the no break case, $\boldsymbol{\gamma}=\mathbf{0}$. As is standard, the lag truncation parameter, $p$, in (3.2) is assumed to satisfy the following condition:

Assumption $\mathcal{B}$. As $T \rightarrow \infty$, the lag truncation parameter $p$ in (3.2) satisfies the condition that $1 / p+p^{3} / T \rightarrow 0$.

In what follows, it is useful to note that when $p=0, D F_{\bar{c}}^{G L S}(\boldsymbol{\tau})$ can be written in the simplified form

$$
\operatorname{DF}_{\bar{c}}^{G L S}(\boldsymbol{\tau})=\frac{\tilde{u}_{T}^{2}-\tilde{u}_{1}^{2}-\sum_{t=2}^{T}\left(\Delta \tilde{u}_{t}\right)^{2}}{2 \sqrt{\hat{\sigma}_{e}^{2} \sum_{t=2}^{T} \tilde{u}_{t-1}^{2}}}
$$

where $\hat{\sigma}_{e}^{2}=(T-2)^{-1} \sum_{t=2}^{T} \hat{e}_{t}^{2}$.

In order to conduct an asymptotic analysis that appropriately mimics the relevant finite sample power properties of unit root tests when uncertainty exists as to the presence of breaks, HLT conduct their asymptotic analysis under a doubly-local setting; that is, in addition to allowing local-to-unity behaviour in the autoregressive root, as above, they also model the trend break magnitudes as localto-zero. Accordingly, in this paper we set the break magnitudes in (2.1) to be $\gamma_{i, T}=\kappa_{i} C(1) \bar{\omega} T^{-1 / 2}$, $i=1, \ldots, m$, where the $\kappa_{i}$ are finite constants, thereby adopting the appropriate Pitman drift for a trend break in a local-to-unit root process. ${ }^{3}$

\section{Asymptotic Behaviour of $M D F_{m}$ under Non-Stationary Volatility}

In this section we establish the large sample properties of the HLT unit root test outlined in the previous section in the case where the volatility process $\sigma_{t}$ is permitted to be generated by any process satisfying Assumption $\mathcal{A}_{3}$.

Theorem 1 Let $y_{t}$ be generated according to (2.1)-(2.4) under $H_{c}$. Let Assumptions $\mathcal{A}$ and $\mathcal{B}$ hold, and let $\gamma_{T}=\kappa C(1) \bar{\omega} T^{-1 / 2}$. Then

$$
M D F_{m} \stackrel{w}{\rightarrow} \inf _{\substack{\tau_{1}, \ldots, \tau_{m} \in \Lambda,\left|\tau_{i}-\tau_{j}\right| \geq \eta, \forall i \neq j}} \mathcal{D}_{c, \bar{c}}\left(\boldsymbol{\tau}_{0}, \boldsymbol{\tau}, \boldsymbol{\kappa}, \eta\right)=: \mathcal{D}_{c, \bar{c}}^{\inf }\left(\boldsymbol{\tau}_{0}, \boldsymbol{\kappa}, \eta\right)
$$

\footnotetext{
${ }^{2}$ We suppress the dependence of quantities such as $\tilde{u}_{t}$ on $\tau$ for notational economy.

${ }^{3}$ Scaling the trend break by $C(1) \bar{\omega}$ is merely a convenience device allowing it to be factored out of the limit distribution of the statistic.
} 
where

$$
\mathcal{D}_{c, \bar{c}}\left(\boldsymbol{\tau}_{0}, \boldsymbol{\tau}, \boldsymbol{\kappa}, \eta\right):=\frac{L_{c, \bar{c}}\left(1, \boldsymbol{\tau}_{0}, \boldsymbol{\tau}, \boldsymbol{\kappa}, \eta\right)^{2}-1}{2 \sqrt{\int_{0}^{1} L_{c, \bar{c}}\left(r, \boldsymbol{\tau}_{0}, \boldsymbol{\tau}, \boldsymbol{\kappa}, \eta\right)^{2} d r}}
$$

with

$$
\begin{aligned}
L_{c, \bar{c}}\left(r, \boldsymbol{\tau}_{0}, \boldsymbol{\tau}, \boldsymbol{\kappa}, \eta\right):= & W_{c}^{\eta}(r)+\boldsymbol{\kappa}^{\prime}\left\{\left(r-\boldsymbol{\tau}_{0}\right) \circ \mathbf{I}_{\boldsymbol{\tau}_{0}}^{r}\right. \\
& -\left[\begin{array}{c}
r \\
(r-\boldsymbol{\tau}) \circ \mathbf{I}_{\boldsymbol{\tau}}^{r}
\end{array}\right]^{\prime}\left[\begin{array}{cc}
a_{\bar{c}} & \mathbf{m}_{\bar{c}}(\boldsymbol{\tau})^{\prime} \\
\mathbf{m}_{\bar{c}}(\boldsymbol{\tau}) & \mathbf{D}_{\bar{c}}(\boldsymbol{\tau})
\end{array}\right]^{-1}\left[\begin{array}{c}
b_{c, \bar{c}, \eta}+\boldsymbol{\kappa}^{\prime} \mathbf{f}_{c, \bar{c}}\left(\boldsymbol{\tau}_{0}\right) \\
\mathbf{b}_{c, \bar{c}, \eta}(\boldsymbol{\tau})+\mathbf{F}_{c, \bar{c}}\left(\boldsymbol{\tau}_{0}, \boldsymbol{\tau}\right) \boldsymbol{\kappa}
\end{array}\right]
\end{aligned}
$$

where

$$
a_{\bar{c}}:=1+\bar{c}+\bar{c}^{2} / 3, \quad b_{c, \bar{c}, \eta}:=(1+\bar{c}) W_{c}^{\eta}(1)+\bar{c}^{2} \int_{0}^{1} s W_{c}^{\eta}(s) d s,
$$

$\mathbf{I}_{\boldsymbol{\tau}_{0}}^{r}$ denotes an $m \times 1$ vector with ith element $\mathbb{I}_{\tau_{0, i}}^{r}, \mathbf{m}_{\bar{c}}(\boldsymbol{\tau}), \mathbf{b}_{c, \bar{c}, \eta}(\boldsymbol{\tau})$ and $\mathbf{f}_{c, \bar{c}}\left(\boldsymbol{\tau}_{0}\right)$ denote $m \times 1$ vectors with ith elements

$$
\begin{aligned}
m_{\bar{c}}\left(\tau_{i}\right):= & a_{\bar{c}}-\tau_{i}\left(1+\bar{c}+\bar{c}^{2} / 2-\bar{c}^{2} \tau_{i}^{2} / 6\right), \\
b_{c, \bar{c}, \eta}\left(\tau_{i}\right):= & \left(1+\bar{c}-\bar{c} \tau_{i}\right) W_{c}^{\eta}(1)-W_{c}^{\eta}\left(\tau_{i}\right)+\bar{c}^{2} \int_{\tau_{i}}^{1}\left(s-\tau_{i}\right) W_{c}^{\eta}(s) d s, \\
f_{c, \bar{c}}\left(\tau_{0, i}\right):= & \left(1-\tau_{0, i}\right)\left\{a_{\bar{c}}-\bar{c}^{2} \tau_{0, i}\left(1+\tau_{0, i}\right) / 6\right\}
\end{aligned}
$$

respectively, $\mathbf{D}_{\bar{c}}(\boldsymbol{\tau})$ and $\mathbf{F}_{c, \bar{c}}\left(\boldsymbol{\tau}_{0}, \boldsymbol{\tau}\right)$ denote $m \times m$ matrices with $i, j$ th elements

$$
\begin{aligned}
d_{\bar{c}}\left(\tau_{i}, \tau_{j}\right):=\quad & \left(1-\tau_{\max }\right)\left\{a_{\bar{c}}+\bar{c} \tau_{\max }-\bar{c}\left(\tau_{i}+\tau_{j}\right)\right. \\
& \left.+\bar{c}^{2}\left(\tau_{\max }+\tau_{\max }^{2}\right) / 3-\bar{c}^{2}\left(\tau_{i}+\tau_{j}\right)\left(1+\tau_{\max }\right) / 2+\bar{c}^{2} \tau_{i} \tau_{j}\right\}, \\
f_{c, \bar{c}}\left(\tau_{0, j}, \tau_{i}\right):=\quad & \left(1-\tau_{0, j}\right)\left\{a_{\bar{c}}-\bar{c} \tau_{i}-\bar{c}^{2} \tau_{i}\left(1-\tau_{0, j}\right) / 2-\bar{c}^{2} \tau_{0, j}\left(1+\tau_{0, j}\right) / 6\right\} \\
& -\left(\tau_{i}-\tau_{0, j}\right)\left\{1-\bar{c}^{2}\left(\tau_{i}-\tau_{0, j}\right)^{2} / 6\right\} \mathbb{I}_{\tau_{0, j}}^{\tau_{i}}
\end{aligned}
$$

respectively, with $\tau_{\max }:=\max \left(\tau_{i}, \tau_{j}\right)$, and where $W_{c}^{\eta}(r):=\int_{0}^{r} e^{-(r-s) c} d W(\eta(s))$, where $W(s)$ is a standard Brownian motion and $\eta(\cdot)$ is the variance profile of the volatility process defined in (2.5).

Remark 3. For the homoskedastic case, HLT propose running the test using asymptotic null critical values obtained from the right member of (4.1) calculated for $c=0$ and $\boldsymbol{\kappa}=\mathbf{0}$. They show numerically that the asymptotic size of the resulting test is conservative when $\boldsymbol{\kappa} \neq \mathbf{0}$ though only ever modestly under-sized.

Remark 4. As can be seen from comparing the representations given in Theorem 1 with the corresponding representations in Theorem 1 of HLT, the asymptotic null distribution of the $M D F_{m}$ statistic is a function of the process $W_{0}^{\eta}(r)$. This distribution reduces to the corresponding distribution given in Theorem 1 of HLT only where the process is homoskedastic, such that $\omega(\cdot)$ is a constant function; here $W_{0}^{\eta}(r)$ reduces to the standard Brownian motion, $W_{0}(r)=W(r)$. It is also clear from the representations in Theorem 1 for $c>0$ that the asymptotic local power functions of the HLT test will also be affected by non-stationary volatility (even if critical values from the correct heteroskedastic null distributions were used) since, as with the null case, it is only where $\omega(\cdot)$ is constant that the representation reduces to the corresponding representation in HLT. 
Remark 5. Notice that the limiting representation in (4.1) depends on the search set, $\Lambda$, just as it does in the homoskedastic case. The asymptotic critical values reported in HLT are appropriate only for $\Lambda=[0.15,0.85]$. An advantage of using a bootstrap implementation of $M D F_{m}$, as we shall propose in section 5 , is that inference can be conducted for any choice of $\Lambda$ without the need for further tables of asymptotic critical values.

To conclude this section we now quantify the impact of a one-time change in volatility on the asymptotic size of the one-break test, $M D F_{1}$. Table 1 reports the asymptotic size of nominal 0.05level $M D F_{1}$ tests, with $\Lambda=[0.15,0.85]$, for a single abrupt shift in volatility from $\sigma_{0}$ to $\sigma_{1}$ at time $\left\lfloor\tau_{\sigma} T\right\rfloor$; i.e. for the volatility function

$$
\omega(s)=\sigma_{0}+\left(\sigma_{1}-\sigma_{0}\right) 1\left(s>\tau_{\sigma}\right), \quad s \in[0,1]
$$

with $\tau_{\sigma} \in[0,1]$. Results are reported for $\sigma_{1} / \sigma_{0} \in\{1 / 10,1 / 5,1 / 2.5,1,2.5,5,10\}$ and $\tau_{\sigma} \in\{0.3,0.5,0.7\}$, allowing for positive and negative breaks in volatility at a range of timings (the setting $\sigma_{1} / \sigma_{0}=1$ giving the constant volatility case). We consider at most a single break in trend at time $\tau_{0,1}=\{0.3,0.5,0.7\}$ (allowing for cases where shift in volatility and the break in trend coincide, and also where their timings differ), with local break magnitudes $\kappa_{1}=\{0,3,6,9,12\}$ ( $\kappa_{1}=0$ representing the no-break case). The sizes reported were computed using direct simulation of the limiting functionals in Theorem 1 , compared with the critical values reported in HLT. We used 50,000 Monte Carlo replications, approximating the Brownian motion processes in the limiting functionals using $N I I D(0,1)$ random variates, with the integrals are approximated by normalized sums of 2,000 steps.

In the homoskedastic case $\left(\sigma_{1} / \sigma_{0}=1\right), M D F_{1}$ has exact size when $\kappa_{1}=0$ since this is precisely the case where the critical values are obtained. For other values of $\kappa_{1}$, it is slightly under-sized, as in HLT. When $\sigma_{1} / \sigma_{0} \neq 1$, however, the shift in volatility virtually always induces an increase in asymptotic size relative to the corresponding homoskedastic case. The upward size distortions increase as $\sigma_{1} / \sigma_{0}$ deviates further from the homoskedastic case of $\sigma_{1} / \sigma_{0}=1$ (for both $\sigma_{1}>\sigma_{0}$ and $\sigma_{1}<\sigma_{0}$ ) for both the no break and local break deterministic specifications. The size distortions are most severe when the timings of the break in trend and the break in volatility are either both early $\left(\tau_{0}=\tau_{\sigma}=0.3\right)$ or both late $\left(\tau_{0}=\tau_{\sigma}=0.7\right)$, with asymptotic size up to around 0.43 in the latter. The impact of a volatility shift on test size is also seen to be strongly dependent on the direction of the shift: when the volatility break occurs early, it is a downward shift that generates relatively greater size distortions than when the shift is upward; conversely, when the volatility break occurs late, it is an upward shift that generates the greater size distortions. It is clear from these results that the presence of heteroskedasticity can have serious implications for the asymptotic size of the HLT unit root test, to the extent that we cannot rely on HLT's tabulated critical values to deliver a size-controlled procedure.

\section{Bootstrap Infimum Tests}

As demonstrated in the previous section, non-stationary volatility introduces a time deformation aspect to the limiting distributions of the HLT unit root statistic, which alters its form vis-à-vis the 
homoskedastic case. In section 5.1 we propose a bootstrap analogue of the $M D F_{m}$ unit root test from section 3. Subsequently in section 5.2 we establish the large sample properties of our proposed bootstrap test. We also discuss in section 5.3 a number of alternative algorithms that might also be expected to display good finite sample properties.

\subsection{The Bootstrap Algorithm}

In this section we present our proposed bootstrap algorithm. Our approach adopts a 'wild bootstrap' scheme (see, inter alia, Liu, 1988, and Mammen, 1993) applied to the second differences of the raw data. This approach, in contrast to standard residual re-sampling schemes used for other bootstrap unit root tests proposed in the literature, replicates the nature of the heteroskedasticity present in the underlying shocks.

The following steps constitute our proposed bootstrap algorithm:

\section{Algorithm 1 (Wild Bootstrap $M D F_{m}$ Test)}

Step 1. Construct the second differences of the data; that is, $\Delta^{2} y_{t}:=\Delta y_{t}-\Delta y_{t-1}, t=3, \ldots, T$.

Step 2. Generate $T$ bootstrap innovations $\varepsilon_{t}^{*}, t=1, \ldots, T$, as follows: $\varepsilon_{t}^{*}:=w_{t} \Delta^{2} y_{t}, t=3, \ldots, T$, and $\varepsilon_{1}^{*}=\varepsilon_{2}^{*}=0$, where $\left\{w_{t}\right\}_{t=1}^{T}$ denotes an independent $N(0,1)$ sequence.

Step 3. Construct the bootstrap sample as the partial sum process defined by

$$
y_{t}^{*}:=\sum_{i=1}^{t} \varepsilon_{i}^{*}, \quad t=1, \ldots, T .
$$

Step 4. Compute the bootstrap test statistic

$$
M D F_{m}^{*}:=\inf _{\substack{\tau_{1}, \ldots, \tau_{m} \in \Lambda,\left|\tau_{i}-\tau_{j}\right| \geq \eta, \forall_{i \neq j}}} D F_{\bar{c}}^{G L S}(\boldsymbol{\tau})^{*}
$$

with

$$
D F_{\bar{c}}^{G L S}(\boldsymbol{\tau})^{*}=\frac{\tilde{u}_{T}^{* 2}-\tilde{u}_{1}^{* 2}-\sum_{t=2}^{T}\left(\Delta \tilde{u}_{t}^{*}\right)^{2}}{2 \sqrt{\hat{\sigma}_{e}^{* 2} \sum_{t=2}^{T} \tilde{u}_{t-1}^{* 2}}}
$$

where $\tilde{u}_{t}^{*}:=y_{t}^{*}-\tilde{\mu}^{*}-\tilde{\beta}^{*} t-\tilde{\boldsymbol{\gamma}}^{* \prime} \mathbf{D} \mathbf{T}_{t}(\boldsymbol{\tau})$, with $\left[\tilde{\mu}^{*}, \tilde{\beta}^{*}, \tilde{\boldsymbol{\gamma}}^{* \prime}\right]^{\prime}$ obtained from a local GLS regression of $\mathbf{y}_{\bar{\rho}}^{*}:=\left[y_{1}^{*}, y_{2}^{*}-\bar{\rho} y_{1}^{*}, \ldots, y_{T}^{*}-\bar{\rho} y_{T-1}^{*}\right]^{\prime}$ on $\mathbf{Z}_{\bar{\rho}, \tau}:=\left[\mathbf{z}_{1}, \mathbf{z}_{2}-\bar{\rho} \mathbf{z}_{1}, \ldots, \mathbf{z}_{T}-\bar{\rho} \mathbf{z}_{T-1}\right]^{\prime}, \mathbf{z}_{t}:=\left[1, t, \mathbf{D} \mathbf{T}_{t}(\boldsymbol{\tau})^{\prime}\right]^{\prime}$ with $\bar{\rho}:=1-\bar{c} / T$. Here $\hat{\sigma}_{e}^{* 2}=(T-2)^{-1} \sum_{t=2}^{T} \hat{e}_{t}^{* 2}$ with $\hat{e}_{t}^{*}$ obtained from the fitted OLS regression

$$
\Delta \tilde{u}_{t}^{*}=\hat{\pi}^{*} \tilde{u}_{t-1}^{*}+\hat{e}_{t}^{*}, \quad t=2, \ldots, T .
$$

Step 5. Bootstrap p-values are computed as: $p_{T}^{*}:=G_{T}^{*}\left(M D F_{m}\right)$, where $G_{T}^{*}(\cdot)$ denotes the conditional (on the original sample data) cumulative density function (cdf) of $M D F_{m}^{*}$. Notice, therefore, that the bootstrap test, run at the $\xi, 0<\xi<1$, significance level, based on $M D F_{m}$, is then defined such that it rejects the unit root null hypothesis, $H_{0}: \rho_{T}=1$ if $p_{T}^{*}<\xi$. 
Remark 6. The second differencing involved in Step 1 of Algorithm 1 removes the effect of the constant and linear trend and reduces each of the trend breaks present to a one-time outlier which will then have no impact in the limit on the behaviour of the resulting bootstrap statistic based on $y_{t}^{*}$, regardless of whether the trend break magnitudes are local-to-zero or fixed. This follows since we can write the scaled $y_{t}^{*}$ as

$$
\begin{aligned}
T^{-1 / 2} y_{\lfloor r T\rfloor}^{*} & =T^{-1 / 2} \sum_{t=3}^{\lfloor r T\rfloor} w_{t} \Delta^{2} u_{t}+T^{-1 / 2} \sum_{j=1}^{m} \gamma_{j} w_{\left\lfloor\tau_{0, j} T\right\rfloor+1} 1\left(\lfloor r T\rfloor>\left\lfloor\tau_{0, j} T\right\rfloor\right) \\
& =T^{-1 / 2} \sum_{t=3}^{\lfloor r T\rfloor} w_{t} \Delta^{2} u_{t}+o_{p}(1)
\end{aligned}
$$

regardless of whether the $\gamma_{j}$ magnitudes are $O\left(T^{-1 / 2}\right)$ or fixed.

Remark 7. As shown in the proof of Theorem 2 below,

$$
T^{-1 / 2} y_{\lfloor r T\rfloor}^{*} \stackrel{w}{\rightarrow}_{p}\left(2 \sum_{i=0}^{\infty} c_{i}\left(c_{i}-c_{i+1}\right)\right)^{1 / 2} \bar{\omega} W_{0}^{\eta}(r)
$$

so we observe that the asymptotic effect of the heteroskedasticity on the bootstrap sample $y_{t}^{*}$ is the same as that on the original raw data $y_{t}$ up to a constant multiple, $\left(2 \sum_{i=0}^{\infty} c_{i}\left(c_{i}-c_{i+1}\right)\right)^{1 / 2}$, which is induced by the second differencing in Step 1; this constant is automatically scaled out of the limit distribution of the bootstrap Dickey-Fuller statistics $D F_{\bar{c}}^{G L S}(\boldsymbol{\tau})^{*}$.

Remark 8. As in Cavaliere and Taylor (2008), the unit root null is imposed on the re-sampling scheme used in Step 3 of Algorithm 1. This has no impact on the power of the bootstrap tests because, conditionally on the original data, the bootstrap innovations $\varepsilon_{t}^{*}$ from Step 2 of Algorithm 1 are serially uncorrelated, allowing us to set the lag length to zero in (5.2). In practice one might also consider adding a sieve-based component to Algorithm 1, of the form outlined in section 3.3 of Cavaliere and Taylor (2009), and selecting the lag length, $p^{*}$ say, in (5.2) as discussed in Cavaliere and Taylor (2009, p.403).

Remark 9. In practice the $\operatorname{cdf} G_{T}^{*}$ will be unknown but can be approximated in the usual way through numerical simulation; see, inter alia, Hansen (1996) and Andrews and Buchinsky (2001). This is done by generating $B$ (conditionally) independent bootstrap statistics, say $M D F_{m, b}^{*}, b=1, \ldots, B$, computed as for $M D F_{m}^{*}$ above but from $y_{b, t}^{*}$ obtained by substituting $\varepsilon_{i}^{*}$ in (5.1) with $\varepsilon_{b, i}^{*}:=\hat{\varepsilon}_{i} w_{b: i}$, again with starting values set to zero, and with $\left\{\left\{w_{b: t}\right\}_{t=1}^{T}\right\}_{b=1}^{B}$ a doubly independent $N(0,1)$ sequence. The simulated bootstrap $p$-value is then computed as $\tilde{p}_{T}^{*}:=B^{-1} \sum_{b=1}^{B} 1\left(M D F_{m, b}^{*} \leq M D F_{m}\right)$, and is such that $\tilde{p}_{T}^{*} \stackrel{a . s .}{\rightarrow} p_{T}^{*}$ as $B \rightarrow \infty$. An approximate standard error for $\tilde{p}_{T}^{*}$ is given by $\left(\tilde{p}_{T}^{*}\left(1-\tilde{p}_{T}^{*}\right) / B\right)^{1 / 2}$; see Hansen (1996, p.419).

\subsection{Asymptotic Properties}

In this section we derive the asymptotic properties of the wild bootstrap unit root test outlined in Algorithm 1 both under the unit root null hypothesis and under near-integrated alternatives.

Our key result is now presented in Theorem 2. Here, for the case of zero or local-to-zero magnitude trend breaks, we show that for any volatility process satisfying Assumption $\mathcal{A}_{3}$ the bootstrap statistics 
from section 5.1 allow us to retrieve asymptotically correct $p$-values appropriate for $\boldsymbol{\kappa}=\mathbf{0}$ under the unit root null.

Theorem 2 Let $y_{t}$ be generated according to (2.1)-(2.4) under $H_{c}$. Let Assumption $\mathcal{A}$ hold, and let $\gamma_{T}=\kappa C(1) \bar{\omega} T^{-1 / 2}$. Then for the bootstrap statistic outlined in Algorithm 1,

$$
M D F_{m}^{*} \stackrel{w}{\rightarrow} p \mathcal{D}_{0, \bar{c}}^{\inf }\left(\boldsymbol{\tau}_{0}, \mathbf{0}, \eta\right)
$$

where $\mathcal{D}_{0, \bar{c}}^{\inf }\left(\boldsymbol{\tau}_{0}, \mathbf{0}, \eta\right)$ is as defined in Theorem 1 , with $c=0$ and $\boldsymbol{\kappa}=\mathbf{0}$. Moreover, if $\rho_{T}=1$, $p_{T}^{*} \stackrel{w}{\rightarrow} U[0,1]$ for $\boldsymbol{\kappa}=\mathbf{0}$.

Theorem 2 demonstrates the usefulness of the wild bootstrap test from Algorithm 1: as the number of observations diverges, the bootstrapped statistic converges to the same null distribution as obtains for the original HLT test statistic when $\boldsymbol{\kappa}=\mathbf{0}$. Notice that $\mathcal{D}_{0, \bar{c}}^{\inf }\left(\boldsymbol{\tau}_{0}, \mathbf{0}, \eta\right)$ does not depend on $\boldsymbol{\tau}_{0}$ when $\boldsymbol{\kappa}=\mathbf{0}$. Consequently, for $\boldsymbol{\kappa}=\mathbf{0}$, the bootstrap $p$-values are uniformly distributed under the null hypothesis, leading to tests with asymptotically correct size. Table 2 reports, for the same settings as those relevant for Table 1 , asymptotic sizes of the bootstrap test $M D F_{1}^{*}$ at the nominal 0.05-level; these results were obtained by simulation of $\mathcal{D}_{c, \bar{c}}^{\inf }\left(\boldsymbol{\tau}_{0}, \boldsymbol{\kappa}, \eta\right)$ in (4.1) with $c=0$, compared with critical values obtained from simulation of $\mathcal{D}_{0, \bar{c}}^{\inf }\left(\boldsymbol{\tau}_{0}, \mathbf{0}, \eta\right)$. In line with Theorem 2 , the asymptotic sizes are exactly 0.05 when $\kappa_{1}=0$, regardless of the nature of the volatility process. This stands in marked contrast to the corresponding entries in Table 1, where the original $M D F_{1}$ was seen to often exhibit severe upward size distortions in the presence of heteroskedasticity. When $\kappa_{1} \neq 0$, the asymptotic size of $M D F_{1}^{*}$ is no longer exactly 0.05 ; this occurs because the bootstrap recovers a critical value for $M D F_{1}$ appropriate only for $\kappa_{1}=0$. Consequently, situations where $M D F_{1}^{*}$ displays under(over-) size correspond to $\kappa_{1} \neq 0$ cases where $M D F_{1}$ had size that was lower (higher) than for the corresponding $\kappa_{1}=0$ case. Notice, however, that whenever upward size distortions do occur, they are only modest in nature, with asymptotic size never exceeding 0.065 ; similarly, much of the under-sizing is also relatively modest.

An additional consequence of the result in Theorem 2 is that the bootstrap $M D F_{m}^{*}$ test shares the same asymptotic local power function as the standard HLT test, $M D F_{m}$, had the $(\boldsymbol{\kappa}=\mathbf{0})$ critical values used for the latter been (infeasibly) adjusted to account for any heteroskedasticity present. In the case where volatility is constant, it also then follows that there is no loss in asymptotic power, relative to using the HLT test, from using the bootstrap $M D F_{m}^{*}$ test from Algorithm 1.

\subsection{Alternative Bootstrap Algorithms}

The bootstrap algorithm for $M D F_{m}^{*}$ given above replicates (asymptotically) the null distribution $\mathcal{D}_{0, \bar{c}}^{\inf }\left(\boldsymbol{\tau}_{0}, \mathbf{0}, \eta\right)$ and, as shown in Table 2, this can lead to a degree of size distortion in the presence of heteroskedasticity when $\boldsymbol{\kappa} \neq \mathbf{0}$. A potential way to alleviate this behaviour is to estimate $\boldsymbol{\tau}_{0}$ and $\gamma$ and incorporate these estimates into the bootstrap data - the intention being that such data will thereby mimic any trend break structure present in the original data. Generalizing the HHLT break 
date estimator to the multiple trend break case, a first differences-based estimator of $\boldsymbol{\tau}_{0}$ is provided by

$$
\tilde{\boldsymbol{\tau}}:=\arg \min _{\substack{\tau_{1}, \ldots, \tau_{m} \in \Lambda, \tau_{i}-\tau_{j} \mid \geq \eta, \forall i \neq j}} T^{-1} \sum_{t=2}^{T} \tilde{v}_{t}(\boldsymbol{\tau})^{2},
$$

where $\tilde{v}_{t}(\boldsymbol{\tau})$ are the residuals from the OLS regression

$$
\Delta y_{t}=\tilde{\beta}+\tilde{\gamma}^{\prime} \Delta \mathbf{D} \mathbf{T}_{t}(\boldsymbol{\tau})+\tilde{v}_{t}(\boldsymbol{\tau})
$$

with the corresponding estimator of $\gamma$ being given by $\tilde{\gamma}=\tilde{\gamma}(\tilde{\boldsymbol{\tau}})$, that is, $\tilde{\gamma}$ evaluated at $\tilde{\boldsymbol{\tau}}$. The drawback of this trend break estimation procedure is that under $H_{0}$ and $H_{c}$, when $\boldsymbol{\kappa}=\mathbf{0}, \boldsymbol{\tau}_{0}$ is unidentified and $\tilde{\boldsymbol{\tau}}$ is then randomly distributed over $\Lambda$. It can also be shown that $\tilde{\gamma}=O_{p}\left(T^{-1 / 2}\right)$ in this case. Therefore, $\tilde{\tau}$ and $\tilde{\gamma}$ will indicate spurious break timings with spurious local break magnitudes of order $T^{-1 / 2}$. In fact, the same is also true for local trend breaks with $\boldsymbol{\kappa} \neq \mathbf{0}$ since $\tilde{\boldsymbol{\tau}}$ is not a consistent estimator of $\boldsymbol{\tau}_{0}$. As a consequence, any bootstrap algorithm that incorporates $\tilde{\boldsymbol{\tau}}$ and $\tilde{\boldsymbol{\gamma}}$ cannot exactly replicate the distribution $\mathcal{D}_{0, \bar{c}}^{\inf }\left(\boldsymbol{\tau}_{0}, \boldsymbol{\kappa}, \eta\right)$ either for $\boldsymbol{\kappa}=\mathbf{0}$ or $\boldsymbol{\kappa} \neq \mathbf{0}$. However, since $\tilde{\boldsymbol{\tau}}$ and $\tilde{\gamma}$ are consistent estimators of $\boldsymbol{\tau}_{0}$ and $\boldsymbol{\gamma}$ in the case when all elements of $\gamma$ are non-zero and of fixed magnitude ${ }^{4}$, pragmatism suggests we might still consider employing them in an alternative bootstrap algorithm to that for $M D F_{m}^{*}$, notwithstanding their theoretical shortcomings in the context of the zero or local trend breaks model. Three such alternatives are now presented, which differ from $M D F_{m}^{*}$ only in how the bootstrap sample $y_{t}^{*}$ is constructed.

(i) $M D F_{m}^{* *}: y_{t}^{*}:=\tilde{\boldsymbol{\gamma}}^{\prime} \mathbf{D} \mathbf{T}_{t}(\tilde{\boldsymbol{\tau}})+\sum_{i=1}^{t} \varepsilon_{i}^{*}, \quad t=1, \ldots, T$.

(ii) $M D F_{m}^{\prime}: y_{t}^{*}:=\sum_{i=2}^{t} w_{t} \tilde{v}_{t}(\tilde{\boldsymbol{\tau}}), \quad t=2, \ldots, T, \quad y_{1}^{*}=0$.

(iii) $M D F_{m}^{\prime \prime}: y_{t}^{*}:=\tilde{\boldsymbol{\gamma}}^{\prime} \mathbf{D T}_{t}(\tilde{\boldsymbol{\tau}})+\sum_{i=2}^{t} w_{t} \tilde{v}_{t}(\tilde{\boldsymbol{\tau}}), \quad t=2, \ldots, T, \quad y_{1}^{*}=0$.

Here $M D F_{m}^{* *}$ is similar to $M D F_{m}^{*}$, but reinstates the broken trend effects in Step 3 of Algorithm 1 using estimates. $M D F_{m}^{\prime}$ and $M D F_{m}^{\prime \prime}$ utilise an alternative approach to removing the broken trend effects in Step 1 of Algorithm 1, with $M D F_{m}^{\prime}$ being an analog of $M D F_{m}^{*}$ but employing the residuals $\tilde{v}_{t}(\tilde{\boldsymbol{\tau}})$ directly instead of second differencing, while $M D F_{m}^{\prime \prime}$ is the corresponding analog of $M D F_{m}^{* *}$.

\section{Finite Sample Simulations}

In this section we investigate the finite sample size and power properties of the original $M D F_{m}$ test and the bootstrap tests $M D F_{m}^{*}, M D F_{m}^{* *}, M D F_{m}^{\prime}$ and $M D F_{m}^{\prime \prime}$ proposed above. For further comparison we also report results for the CKP test, which we denote by $C K P_{m}$, where $m$ corresponds to the maximum number of breaks considered in the procedure. In line with our asymptotic results we set $\Lambda=[0.15,0.85]$ for the implementation of all tests, and also set the separation fraction between consecutive breaks to be $\eta=0.15$. We abstract from the effects of serial correlation, generating $z_{t} \sim$

\footnotetext{
${ }^{4}$ The proof of this follows straightforwardly from the consistency proof given for the single break case in Cavaliere et al. (2011).
} 
$\operatorname{NIID}(0,1)$ and $C(L)=1$, and setting $p=0$ in the Dickey-Fuller regressions, and we also make the corresponding setting when constructing the $C K P_{m}$ statistics. In line with the local trend break specification adopted in the large sample analysis, we set $\gamma_{T}=\kappa \bar{\omega}_{T} T^{-1 / 2}$ with $\bar{\omega}_{T}:=\sqrt{T^{-1} \sum_{t=1}^{T} \sigma_{t}^{2}}$. All simulations were conducted at the nominal 0.05 level using 1,000 Monte Carlo replications and $B=499$ bootstrap replications. In sections 6.1 and 6.2 below, we present results for finite sample size and power, respectively.

\subsection{Size}

Tables 3 and 4 presents size results for $C K P_{1}, M D F_{1}$ and its corresponding bootstrap variants for a single break in trend and a single shift in volatility, with sample sizes $T=150$ and $T=300$ respectively. We set $\mu=\beta=0$ without loss of generality, and here consider $c=0$ (i.e. $\rho_{T}=1$ ) with $u_{1}=\varepsilon_{1}$. We focus on the cases of $\tau_{0,1}=\{0.5,0.7\}$ and $\tau_{\sigma}=\{0.5,0.7\}$ with $\sigma_{1} / \sigma_{0} \in\{1,2.5,5,10\}$ (we let $\sigma_{0}=1$ without loss of generality), again setting $\kappa_{1}=\{0,3,6,9,12\}$; these representative combinations are chosen so as to capture the pertinent features observed in the asymptotic results in Tables 1 and 2 .

The finite sample size behaviour of $M D F_{1}$ is seen to closely mirror the patterns of asymptotic size observed in Table 1, with the numerical values of the sizes when $T=300$ bearing a close resemblance to the corresponding asymptotic sizes across all settings. In particular, the test is most over-sized either when no trend break occurs and $\tau_{\sigma}=0.7$, or a trend break is present and $\tau_{0}=\tau_{\sigma}=0.7$, with the distortions again at their most severe for the larger values of $\sigma_{1} / \sigma_{0}$. The sizes of $C K P_{1}$ follow the same pattern as those of $M D F_{1}$, with severe upward size distortions of a similar magnitude observed in the worst cases. In contrast, the bootstrap test $M D F_{1}^{*}$ never displays any serious over-size across the various deterministic and volatility settings considered, although it can be under-sized when both a trend break and volatility change are present (in line with the limit results of Table 2). As regards the alternative bootstrap approach $M D F_{1}^{* *}$, we see that much of the under-size associated with $M D F_{1}^{*}$ is ameliorated through the reinstatement of the estimated break in the bootstrap samples, unless $\kappa_{1}$ is small (since here the break date and magnitude cannot be reliably estimated). Moreover, despite not exactly replicating the null distribution asymptotically in the no-break case, $M D F_{1}^{* *}$ retains good size control in this case also; indeed, it is typically slightly closer to nominal size than $M D F_{1}^{*}$. Lastly, we observe that $M D F_{1}^{\prime}$ and $M D F_{1}^{\prime \prime}$ display much the same size patterns as $M D F_{1}^{*}$ and $M D F_{1}^{* *}$, respectively, although they tend to have somewhat greater size than the corresponding second difference-based bootstrap procedures.

We now consider size simulations for a DGP involving two breaks in trend and a single shift in volatility. Tables 5 and 6 report results for $C K P_{2}, M D F_{2}, M D F_{2}^{*}, M D F_{2}^{* *}, M D F_{2}^{\prime}$ and $M D F_{2}^{\prime \prime}$ for $T=$ 150 and $T=300$, respectively, with trend break timings $\tau_{0,1}=0.3, \tau_{0,2}=0.5$ and $\tau_{0,1}=0.5, \tau_{0,2}=0.7$ with magnitudes $\kappa_{1}=-\kappa, \kappa_{2}=\kappa$ where $\kappa=\{0,6,12\}$, and volatility settings $\tau_{\sigma}=\{0.5,0.7\}$ with $\sigma_{1} / \sigma_{0} \in\{1,5,10\}$. For $M D F_{2}$, we observe over-size in the presence of heteroskedasticity, this being particularly acute either when no trend breaks occur, or when trend breaks occur with the later set of break timings $\left(\tau_{0,1}=0.5, \tau_{0,2}=0.7\right)$. In line with the results above, the size distortions are greatest 
for later changes in volatility. $C K P_{2}$ also suffers from severe over-size in the case of no trend breaks when $\sigma_{1} / \sigma_{0}>1$, although when the trend breaks are non-zero, it becomes quite under-sized in many cases; this latter feature arises because the break detection methodology implicit in the CKP approach is ineffective in the presence of opposite signed local breaks of moderate magnitude (see HLT for more details). As in the single trend break case, $M D F_{2}^{*}$ and $M D F_{2}^{* *}$ are not subject to serious over-size, and while the former can again be under-sized in the presence of both trend breaks and volatility change, this under-size is considerably reduced by $M D F_{2}^{* *}$. As before, size results for $M D F_{2}^{\prime}$ and $M D F_{2}^{\prime \prime}$ bear a broad resemblance to those for $M D F_{2}^{*}$ and $M D F_{2}^{* *}$, respectively, but again the sizes can be a little inflated.

\subsection{Power}

Tables 7 and 8 report finite sample powers for $T=150$ for the same settings as employed in Table 3 , for $c=20$ and $c=30$, respectively. In addition to presenting raw powers for all tests (including those for $C K P_{1}$ and $M D F_{1}$ for completeness despite their lack of size control), we also report two critical valueadjusted versions of $M D F_{1}$ to aid comparison with the bootstrap procedures - a size-adjusted version that uses the appropriate 0.05 -level critical value for $M D F_{1}$ for each volatility process and local break magnitude setting (denoted $M D F_{1}^{a d j}$ in the tables) and also a partially adjusted version that retains use of $\boldsymbol{\kappa}=\mathbf{0}$ critical values but adjusts those critical values for the effects of any heteroskedasticity present (denoted $M D F_{1}^{h a d j}$ ). Given that $M D F_{1}$ was seen in Table 3 to always have finite sample size in excess of nominal size (and sometimes dramatically so), the powers of both $M D F_{1}^{a d j}$ and $M D F_{1}^{\text {hadj }}$ are lower than their raw power counterparts throughout. We observe that the powers of $M D F_{1}^{*}$ and $M D F_{1}^{h a d j}$ are generally quite similar, as we would expect in light of Theorem 2 and our associated discussion. Also unsurprisingly, the powers of $M D F_{1}^{*}$ are almost identical to those of $M D F_{1}^{\text {adj }}$ when $\kappa_{1}=0$. In those $\kappa_{1} \neq 0$ cases where $M D F_{1}^{*}$ is under-sized, it is seen to lose power compared to $M D F_{1}^{a d j}$, and while the losses can be up to 0.25 (for $c=30$ ), they are typically rather more modest. As we might expect, $M D F_{1}^{* *}$ makes up most of these relative power losses, having power close to that of $M D F_{1}^{a d j}$ in most cases, the exception being when $\kappa_{1}$ is small (where $M D F_{1}^{* *}$ was seen to remain under-sized). The powers of $M D F_{1}^{\prime}$ and $M D F_{1}^{\prime \prime}$ are of course similar to (a little greater than) $M D F_{1}^{*}$ and $M D F_{1}^{* *}$, respectively, in line with the corresponding size behaviour of these procedures.

Finally, Table 9 presents powers for $T=150$ in the case of two breaks in trend, for the same settings as in Table 5, for $c=30$. Notice that $C K P_{2}$ (and to a somewhat lesser extent $C K P_{1}$ in Tables 7 and 8) displays the well-documented phenomenon of extremely low power for intermediate local break magnitudes, caused by low break detection rates in these circumstances; see, inter alia, HLT. As in the single trend break case, the powers of $M D F_{2}^{*}$ are very close to those of $M D F_{2}^{\text {hadj }}$. In addition, the $M D F_{2}^{*}$ powers are similar to those of $M D F_{2}^{a d j}$ when $\kappa=0$, but they typically fall below these levels when $\kappa \neq 0$. However, $M D F_{2}^{* *}$ is again seen to recover most of these losses relative to $M D F_{2}^{a d j}$. Once more, $M D F_{2}^{\prime}$ and $M D F_{2}^{\prime \prime}$ have similar levels of power to $M D F_{1}^{*}$ and $M D F_{1}^{* *}$, respectively. 


\section{Conclusions}

In this paper we have explored the impact that non-stationary volatility has on the infimum DickeyFuller-type test proposed in Harvey et al. (2013) which allows for multiple possible breaks in trend. Numerical evidence was presented which showed that non-stationary volatility can have potentially serious implications for the reliability of this test with size often being very substantially above the nominal level. This was shown to be a feature of the limiting distributions of the statistic. To help rectify this problem, we have proposed wild bootstrap-based implementations of the Harvey et al. (2013) test, this approach having proved to be highly successful in other unit root testing applications. The proposed bootstrap tests have the considerable advantage that they are not tied to a given parametric model of volatility within the class of non-stationary volatility processes considered. The asymptotic effectiveness of our proposed bootstrap tests within the class of non-stationary volatility considered was demonstrated. Monte Carlo simulation evidence for the case of a one-time change in volatility for models with both a single break in trend and a double break in trend was also reported which suggested that the proposed bootstrap unit root tests perform well in finite samples avoiding the large over-size problems that can occur with the Harvey et al.'s (2013) test, yet emulating the finite sample power properties of (infeasible) critical value-adjusted implementations of their test. In future work, it would be interesting to explore the possibility of analagous procedures for GLS detrended variants of Im et al. (2003)-type panel unit root tests where breaks in trend and non-stationary volatility are potentially an issue for each series.

\section{A Appendix}

Proof of Theorem 1. For expositional brevity we demonstrate the result of Theorem 1 in the serially uncorrelated case $\varepsilon_{t}=e_{t}$ (i.e. $C(L)=1$ ), setting $p=0$ in (3.2) accordingly. The result continues to hold under the more general conditions for $\varepsilon_{t}$ of Assumption 1, provided Assumption $\mathcal{B}$ holds. In what follows, we also set $\mu=\beta=0$ without loss of generality. We will make use of the following weak convergence results, which follow from straightforward extensions of the results in Cavaliere and Taylor (2007):

$$
\begin{aligned}
& T^{-1 / 2} u_{\lfloor r T\rfloor} \stackrel{w}{\rightarrow} \bar{\omega} W_{c}^{\eta}(r) \\
& T^{-3 / 2} \sum_{t=\lfloor r T\rfloor}^{T} u_{t-1} \stackrel{w}{\rightarrow} \bar{\omega} \int_{r}^{1} W_{c}^{\eta}(s) d s \\
& T^{-3 / 2} \sum_{t=\lfloor r T\rfloor}^{T} t \Delta u_{t} \stackrel{w}{\rightarrow} \bar{\omega}\left\{W_{c}^{\eta}(1)-r W_{c}^{\eta}(r)-\int_{r}^{1} W_{c}^{\eta}(s) d s\right\} \\
& T^{-5 / 2} \sum_{t=\lfloor r T\rfloor}^{T} t u_{t-1} \stackrel{w}{\rightarrow} \bar{\omega} \int_{r}^{1} s W_{c}^{\eta}(s) d s .
\end{aligned}
$$

First, for any $\tau_{1}, \ldots, \tau_{m} \in \Lambda$, consider the estimators $\tilde{\mu}, \tilde{\beta}$ and $\tilde{\gamma}$. Following HLT we find

$$
\left[\begin{array}{c}
\tilde{\mu} \\
T^{1 / 2} \tilde{\beta} \\
T^{1 / 2} \tilde{\boldsymbol{\gamma}}
\end{array}\right] \stackrel{w}{\rightarrow}\left[\begin{array}{ccc}
1 & 0 & \mathbf{0}^{\prime} \\
0 & a_{\bar{c}} & \mathbf{m}_{\bar{c}}(\boldsymbol{\tau})^{\prime} \\
\mathbf{0} & \mathbf{m}_{\bar{c}}(\boldsymbol{\tau}) & \mathbf{D}_{\bar{c}}(\boldsymbol{\tau})
\end{array}\right]^{-1} \times \lim _{T \rightarrow \infty}\left[\begin{array}{c}
h_{1}^{y} \\
T^{-1 / 2} h_{2}^{y} \\
T^{-1 / 2} \mathbf{p}
\end{array}\right]
$$


where

$$
\begin{array}{ll}
h_{1}^{y}:= & y_{1}+(1-\bar{\rho}) \sum_{t=2}^{T}\left(y_{t}-\bar{\rho} y_{t-1}\right), \\
h_{2}^{y}:= & y_{1}+\sum_{t=2}^{T}\left(y_{t}-\bar{\rho} y_{t-1}\right)\{t-\bar{\rho}(t-1)\}
\end{array}
$$

and where the $(m \times 1)$ vector $\mathbf{p}$ has $i$ th element

$$
p_{i}:=\sum_{t=\left\lfloor\tau_{i} T\right\rfloor+1}^{T}\left(y_{t}-\bar{\rho} y_{t-1}\right)\left\{t-\left\lfloor\tau_{i} T\right\rfloor-\bar{\rho}\left(t-\left\lfloor\tau_{i} T\right\rfloor-1\right)\right\} .
$$

For the limits of $h_{1}^{y}, h_{2}^{y}$ and the elements of $\mathbf{p}$ we obtain, using (A.1)-(A.4), and on defining $D U_{t}\left(\tau_{0, i}\right):=$ $\Delta D T_{t}\left(\tau_{0, i}\right)$,

$$
\begin{aligned}
& h_{1}^{y}=y_{1}+\bar{c} T^{-1}\left(y_{T}-y_{1}\right)+\bar{c}^{2} T^{-2} \sum_{t=2}^{T} y_{t-1}=u_{1}+o_{p}(1), \\
& T^{-1 / 2} h_{2}^{y}=\bar{c} T^{-3 / 2} \sum_{t=2}^{T} t \Delta u_{t}+T^{-1 / 2} u_{T}+\bar{c}^{2} T^{-5 / 2} \sum_{t=2}^{T} t u_{t-1}+\bar{c} T^{-3 / 2} \sum_{t=2}^{T} u_{t-1} \\
& +\bar{\omega} \bar{c} \sum_{i=1}^{m} \kappa_{i} T^{-2} \sum_{t=2}^{T} t D U_{t}\left(\tau_{0, i}\right)+\bar{\omega} \sum_{i=1}^{m} \kappa_{i} T^{-1}\left(T-\left\lfloor\tau_{0, i} T\right\rfloor\right) \\
& +\bar{\omega} \bar{c}^{2} \sum_{i=1}^{m} \kappa_{i} T^{-3} \sum_{t=2}^{T} t D T_{t-1}\left(\tau_{0, i}\right)+\bar{\omega} \bar{c} \sum_{i=1}^{m} \kappa_{i} T^{-2} \sum_{t=2}^{T} D T_{t-1}\left(\tau_{0, i}\right)+o_{p}(1) \\
& \stackrel{w}{\rightarrow} \bar{c}\left\{\bar{\omega} W_{c}^{\eta}(1)-\bar{\omega} \int_{0}^{1} W_{c}^{\eta}(s) d s\right\}+\bar{\omega} W_{c}^{\eta}(1)+\bar{c}^{2} \bar{\omega} \int_{0}^{1} s W_{c}^{\eta}(s) d s+\bar{c} \bar{\omega} \int_{0}^{1} W_{c}^{\eta}(s) d s \\
& +\bar{\omega} \bar{c} \sum_{i=1}^{m} \kappa_{i}\left(1-\tau_{0, i}^{2}\right) / 2+\bar{\omega} \sum_{i=1}^{m} \kappa_{i}\left(1-\tau_{0, i}\right) \\
& +\bar{\omega} \bar{c}^{2} \sum_{i=1}^{m} \kappa_{i}\left\{\left(1-\tau_{0, i}^{3}\right) / 3-\tau_{0, i}\left(1-\tau_{0, i}^{2}\right) / 2\right\}+\bar{\omega} \bar{c} \sum_{i=1}^{m} \kappa_{i}\left(1-\tau_{0, i}\right)^{2} / 2 \\
& =\bar{\omega}\left\{b_{c, \bar{c}, \eta}+\boldsymbol{\kappa}^{\prime} \mathbf{f}_{c, \bar{c}}\left(\boldsymbol{\tau}_{0}\right)\right\} \text {, } \\
& T^{-1 / 2} p_{i}=T^{-1 / 2} u_{T}-T^{-1 / 2} u_{\left\lfloor\tau_{i} T\right\rfloor}+\bar{c} T^{-3 / 2} \sum_{t=\left\lfloor\tau_{i} T\right\rfloor+1}^{T} t \Delta u_{t}-\bar{c} \tau_{i} T^{-1 / 2} \sum_{t=\left\lfloor\tau_{i} T\right\rfloor+1}^{T} \Delta u_{t} \\
& +\bar{c} T^{-3 / 2} \sum_{t=\left\lfloor\tau_{i} T\right\rfloor+1}^{T} u_{t-1}+\bar{c}^{2} T^{-5 / 2} \sum_{t=\left\lfloor\tau_{i} T\right\rfloor+1}^{T} t u_{t-1}-\bar{c}^{2} \tau_{i} T^{-3 / 2} \sum_{t=\left\lfloor\tau_{i} T\right\rfloor+1}^{T} u_{t-1} \\
& +\bar{\omega} \sum_{j=1}^{m} \kappa_{j}\left(1-\tau_{0, j}\right)-\bar{\omega} \sum_{j=1}^{m} \kappa_{j}\left(\tau_{i}-\tau_{0, j}\right) \mathbb{I}_{\tau_{0, j}}^{\tau_{i}}+\bar{\omega} \bar{c} \sum_{j=1}^{m} \kappa_{j} T^{-2} \sum_{t=\left\lfloor\tau_{i} T\right\rfloor+1}^{T} t D U_{t}\left(\tau_{0, j}\right) \\
& -\bar{\omega} \bar{c} \sum_{j=1}^{m} \kappa_{j} \tau_{i} T^{-1} \sum_{t=\left\lfloor\tau_{i} T\right\rfloor+1}^{T} D U_{t-1}\left(\tau_{0, j}\right)+\bar{\omega} \bar{c} \sum_{j=1}^{m} \kappa_{j} T^{-2} \sum_{t=\left\lfloor\tau_{i} T\right\rfloor+1}^{T} D T_{t-1}\left(\tau_{0, j}\right) \\
& +\bar{\omega} \bar{c}^{2} \sum_{j=1}^{m} \kappa_{j} T^{-3} \sum_{t=\left\lfloor\tau_{i} T\right\rfloor+1}^{T} t D T_{t-1}\left(\tau_{0, j}\right)-\bar{\omega} \bar{c}^{2} \sum_{j=1}^{m} \kappa_{j} \tau_{i} T^{-2} \sum_{t=\left\lfloor\tau_{i} T\right\rfloor+1}^{T} D T_{t-1}\left(\tau_{0, j}\right)+o_{p}(1) \\
& \stackrel{w}{\rightarrow} \bar{\omega} W_{c}^{\eta}(1)-\bar{\omega} W_{c}^{\eta}\left(\tau_{i}\right)+\bar{\omega} \bar{c}\left\{W_{c}^{\eta}(1)-\tau_{i} W_{c}^{\eta}\left(\tau_{i}\right)-\int_{\tau_{i}}^{1} W_{c}^{\eta}(s) d s\right\}-\bar{\omega} \bar{c} \tau_{i}\left\{W_{c}^{\eta}(1)-W_{c}^{\eta}\left(\tau_{i}\right)\right\} \\
& +\bar{c} \bar{\omega} \int_{\tau_{i}}^{1} W_{c}^{\eta}(s) d s+\bar{c}^{2} \bar{\omega} \int_{\tau_{i}}^{1} s W_{c}^{\eta}(s) d s-\bar{c}^{2} \tau_{i} \bar{\omega} \int_{\tau_{i}}^{1} W_{c}^{\eta}(s) d s \\
& +\bar{\omega} \sum_{j=1}^{m} \kappa_{j}\left(1-\tau_{0, j}\right)-\bar{\omega} \sum_{j=1}^{m} \kappa_{j}\left(\tau_{i}-\tau_{0, j}\right) \mathbb{I}_{\tau_{0, j}}^{\tau_{i}} \\
& +\bar{\omega} \bar{c} \sum_{j=1}^{m} \kappa_{j}\left\{\left(1-\tau_{0, j}^{2}\right) / 2-\left(\tau_{i}^{2}-\tau_{0, j}^{2}\right) \mathbb{I}_{\tau_{0, j}}^{\tau_{i}} / 2\right\}-\bar{\omega} \bar{c} \sum_{j=1}^{m} \kappa_{j} \tau_{i}\left\{1-\tau_{0, j}-\left(\tau_{i}-\tau_{0, j}\right) \mathbb{I}_{\tau_{0, j}}^{\tau_{i}}\right\} \\
& +\bar{\omega} \bar{c} \sum_{j=1}^{m} \kappa_{j}\left(1-\bar{c} \tau_{i}\right)\left\{\left(1-\tau_{0, j}\right)^{2} / 2-\left(\tau_{i}-\tau_{0, j}\right)^{2} \mathbb{I}_{\tau_{0, j}}^{\tau_{i}} / 2\right\} \\
& +\bar{\omega} \bar{c}^{2} \sum_{j=1}^{m} \kappa_{j}\left[\left(1-\tau_{0, j}^{3}\right) / 3-\tau_{0, j}\left(1-\tau_{0, j}^{2}\right) / 2-\left\{\left(\tau_{i}^{3}-\tau_{0, j}^{3}\right) / 3-\tau_{0, j}\left(\tau_{i}^{2}-\tau_{0, j}^{2}\right) / 2\right\} \mathbb{I}_{\tau_{0, j}}^{\tau_{i}}\right] \\
& =\bar{\omega}\left\{b_{c, \bar{c}, \eta}\left(\tau_{i}\right)+\sum_{j=1}^{m} \kappa_{j} f_{c, \bar{c}}\left(\tau_{0, j}, \tau_{i}\right)\right\} \text {, }
\end{aligned}
$$

where (A.5) and (A.6) follow upon simplification after gathering terms.

We therefore have that

$$
\left[\begin{array}{c}
\tilde{\mu} \\
T^{1 / 2} \tilde{\beta} \\
T^{1 / 2} \tilde{\boldsymbol{\gamma}}
\end{array}\right] \stackrel{w}{\rightarrow}\left[\begin{array}{ccc}
1 & 0 & \mathbf{0}^{\prime} \\
0 & a_{\bar{c}} & \mathbf{m}_{\bar{c}}(\boldsymbol{\tau})^{\prime} \\
\mathbf{0} & \mathbf{m}_{\bar{c}}(\boldsymbol{\tau}) & \mathbf{D}_{\bar{c}}(\boldsymbol{\tau})
\end{array}\right]^{-1}\left[\begin{array}{c}
u_{1} \\
\bar{\omega}\left\{b_{c, \bar{c}, \eta}+\boldsymbol{\kappa}^{\prime} \mathbf{f}_{c, \bar{c}}\left(\boldsymbol{\tau}_{0}\right)\right\} \\
\bar{\omega}\left\{\mathbf{b}_{c, \bar{c}, \eta}(\boldsymbol{\tau})+\mathbf{F}_{c, \bar{c}}\left(\boldsymbol{\tau}_{0}, \boldsymbol{\tau}\right) \boldsymbol{\kappa}\right\}
\end{array}\right]
$$


which gives the limit of $T^{-1 / 2} \tilde{u}_{\lfloor r T\rfloor}$ as

$$
\begin{aligned}
T^{-1 / 2} \tilde{u}_{\lfloor r T\rfloor}= & T^{-1 / 2} y_{\lfloor r T\rfloor}-T^{-1 / 2} \tilde{\mu}-T^{-1 / 2} \tilde{\beta}\lfloor r T\rfloor-T^{-1 / 2} \tilde{\boldsymbol{\gamma}}^{\prime}\left\{(\lfloor r T\rfloor-\lfloor\boldsymbol{\tau} T\rfloor) \circ \mathbf{I}_{\boldsymbol{\tau}}^{r}\right\} \\
= & T^{-1 / 2} u_{\lfloor r T\rfloor}+\bar{\omega} \boldsymbol{\kappa}^{\prime}\left\{\left(r-\boldsymbol{\tau}_{0}\right) \circ \mathbf{I}_{\boldsymbol{\tau}_{0}}^{r}\right\}-\left[\begin{array}{c}
r \\
(r-\boldsymbol{\tau}) \circ \mathbf{I}_{\boldsymbol{\tau}}^{r}
\end{array}\right]^{\prime}\left[\begin{array}{c}
T^{1 / 2} \tilde{\beta} \\
T^{1 / 2} \tilde{\boldsymbol{\gamma}}
\end{array}\right]+o_{p}(1) \\
& \stackrel{w}{\rightarrow} \bar{\omega} W_{c}^{\eta}(r)+\bar{\omega} \boldsymbol{\kappa}^{\prime}\left\{\left(r-\boldsymbol{\tau}_{0}\right) \circ \mathbf{I}_{\boldsymbol{\tau}_{0}}^{r}\right\} \\
& -\left[\begin{array}{c}
r \\
(r-\boldsymbol{\tau}) \circ \mathbf{I}_{\boldsymbol{\tau}}^{r}
\end{array}\right]^{\prime}\left[\begin{array}{cc}
a_{\bar{c}} & \mathbf{m}_{\bar{c}}(\boldsymbol{\tau})^{\prime} \\
\mathbf{m}_{\bar{c}}(\boldsymbol{\tau}) & \mathbf{D}_{\bar{c}}(\boldsymbol{\tau})
\end{array}\right]^{-1}\left[\begin{array}{c}
\bar{\omega}\left\{b_{c, \bar{c}, \eta}+\boldsymbol{\kappa}^{\prime} \mathbf{f}_{c, \bar{c}}\left(\boldsymbol{\tau}_{0}\right)\right\} \\
\bar{\omega}\left\{\mathbf{b}_{c, \bar{c}, \eta}(\boldsymbol{\tau})+\mathbf{F}_{c, \bar{c}}\left(\boldsymbol{\tau}_{0}, \boldsymbol{\tau}\right) \boldsymbol{\kappa}\right\}
\end{array}\right] \\
= & \bar{\omega} L_{c, \bar{c}}\left(r, \boldsymbol{\tau}_{0}, \boldsymbol{\tau}, \boldsymbol{\kappa}\right) .
\end{aligned}
$$

Next,

$$
\begin{aligned}
\hat{\sigma}_{e}^{2} & =(T-2)^{-1} \sum_{t=2}^{T} \hat{e}_{t}^{2} \\
& =T^{-1} \sum_{t=2}^{T}\left(\Delta \tilde{u}_{t}\right)^{2}+o_{p}(1) \\
& =T^{-1} \sum_{t=2}^{T} \varepsilon_{t}^{2}+o_{p}(1) \stackrel{p}{\rightarrow} \bar{\omega}^{2}
\end{aligned}
$$

Given $p=0$, write $D F_{\bar{c}}^{G L S}(\boldsymbol{\tau})$ in the form

$$
\begin{aligned}
D F_{\bar{c}}^{G L S}(\boldsymbol{\tau})= & \frac{\tilde{u}_{T}^{2}-\tilde{u}_{1}^{2}-\sum_{t=2}^{T}\left(\Delta \tilde{u}_{t}\right)^{2}}{2 \sqrt{\hat{\sigma}_{e}^{2} \sum_{t=2}^{T} \tilde{u}_{t-1}^{2}}} \\
= & \frac{\left(T^{-1 / 2} \tilde{u}_{T}\right)^{2}-T^{-1} \sum_{t=2}^{T}\left(\Delta \tilde{u}_{t}\right)^{2}}{2 \sqrt{\hat{\sigma}_{e}^{2} T^{-2} \sum_{t=2}^{T} \tilde{u}_{t-1}^{2}}}+o_{p}(1) \\
& \stackrel{w}{\rightarrow} \frac{\bar{\omega}^{2} L_{c, \bar{c}}\left(1, \boldsymbol{\tau}_{0}, \boldsymbol{\tau}, \boldsymbol{\kappa}, \eta\right)^{2}-\bar{\omega}^{2}}{2 \sqrt{\bar{\omega}^{2} \cdot \bar{\omega}^{2} \int_{0}^{1} L_{c, \bar{c}}\left(r, \boldsymbol{\tau}_{0}, \boldsymbol{\tau}, \boldsymbol{\kappa}, \eta\right)^{2} d r}}=\mathcal{D}_{c, \bar{c}}\left(\boldsymbol{\tau}_{0}, \boldsymbol{\tau}, \boldsymbol{\kappa}, \eta\right) .
\end{aligned}
$$

The stated result for $M D F_{m}$ then follows from this fixed $\boldsymbol{\tau}$ representation, using the relevant arguments proved in Zivot and Andrews (1992) and an application of the continuous mapping theorem $[\mathrm{CMT}]$.

Proof of Theorem 2. In what follows, we again set $\mu=\beta=0$ with no loss of generality. Throughout the proof of Theorem 2, we use $\iota_{k}$ to denote the $k \times 1$ unit vector, and $P^{*}$ and $E^{*}$ to denote respectively the probability and expectation conditional on the realization of original sample. Moreover, for a given sequence $X_{T}^{*}$ computed on the bootstrap data, the notation $X_{T}^{*}=o_{p}^{*}(1)$ is taken to mean that $P^{*}\left(\left|X_{T}^{*}\right|>\epsilon\right) \rightarrow 0$ in probability for any $\epsilon>0$ as $T \rightarrow \infty$.

According to Algorithm 1, we have that

$$
T^{-1 / 2} y_{\lfloor r T\rfloor}^{*}:=T^{-1 / 2} \sum_{t=1}^{\lfloor r T\rfloor} \varepsilon_{t}^{*}=T^{-1 / 2} \sum_{t=3}^{\lfloor r T\rfloor} w_{t} \Delta^{2} y_{t} .
$$


Since $w_{t}$ is independent $N(0,1)$, we have that, conditionally on the original sample,

$$
T^{-1 / 2} y_{\lfloor r T\rfloor}^{*} \sim N\left(0, V_{T}(r)\right)
$$

with $V_{T}(r):=T^{-1} \sum_{t=3}^{\lfloor r T\rfloor}\left(\Delta^{2} y_{t}\right)^{2}$. As in Cavaliere and Taylor (2008), if

$$
V_{T}(r) \stackrel{p}{\rightarrow} \kappa^{2} \int_{0}^{r} \omega(s)^{2} d s
$$

pointwise for some positive constant $\kappa$ (independent of $r$ ) then it holds that

$$
T^{-1 / 2} y_{\lfloor\cdot T\rfloor}^{*} \stackrel{w}{\rightarrow} p \bar{\omega}^{*} W_{0}^{\eta}(\cdot)
$$

where $\bar{\omega}^{*}:=\kappa\left(\int_{0}^{1} \omega(s)^{2} d s\right)^{1 / 2}$ and $W_{0}^{\eta}(r):=\int_{0}^{r} d B(\eta(s))$ is a variance-transformed Brownian motion with variance profile $\eta(r):=\left(\int_{0}^{1} \omega(s)^{2} d s\right)^{-1} \int_{0}^{r} \omega(s)^{2} d s$. To show that (A.7) holds, first notice that $\Delta^{2} y_{t}=\gamma_{T}^{\prime} \Delta^{2} \mathbf{D T}_{t}\left(\tau_{0}\right)+\Delta^{2} u_{t}$, which implies that $V_{T}(r)$ can be written as

$$
V_{T}(r)=T^{-1} \sum_{t=3}^{\lfloor r T\rfloor}\left(\Delta^{2} u_{t}\right)^{2}+T^{-1} \sum_{t=3}^{\lfloor r T\rfloor}\left(\gamma_{T}^{\prime} \Delta^{2} \mathbf{D} \mathbf{T}_{t}\left(\tau_{0}\right)\right)^{2}+2 T^{-1} \sum_{t=3}^{\lfloor r T\rfloor}\left(\Delta^{2} u_{t}\right)\left(\gamma_{T}^{\prime} \Delta^{2} \mathbf{D} \mathbf{T}_{t}\left(\tau_{0}\right)\right)
$$

where $\Delta^{2} \mathbf{D} \mathbf{T}_{t}\left(\tau_{0}\right)$ is a vector of $m$ impulse dummies, i.e. its $i$-th element is given by $\mathbb{I}\left(t=\left\lfloor\tau_{i} T\right\rfloor+1\right)$. Hence, as $\gamma_{T}=O\left(T^{-1 / 2}\right)$ we have that

$$
\sup _{r \in[0,1]} T^{-1} \sum_{t=3}^{\lfloor r T\rfloor}\left(\gamma_{T}^{\prime} \Delta^{2} \mathbf{D} \mathbf{T}_{t}\left(\tau_{0}\right)\right)^{2} \leq T^{-1} \sum_{t=3}^{T}\left(\gamma_{T}^{\prime} \Delta^{2} \mathbf{D} \mathbf{T}_{t}\left(\tau_{0}\right)\right)^{2}=T^{-1}\left(\gamma_{T}^{\prime} \boldsymbol{\gamma}_{T}\right)=O\left(T^{-2}\right) .
$$

Similarly, we have that

$$
\begin{aligned}
\sup _{r \in[0,1]}\left|T^{-1} \sum_{t=3}^{\lfloor r T\rfloor}\left(\Delta^{2} u_{t}\right)\left(\gamma_{T}^{\prime} \Delta^{2} \mathbf{D} \mathbf{T}_{t}\left(\tau_{0}\right)\right)\right| & \leq T^{-1} \sum_{t=3}^{T}\left|\Delta^{2} u_{t}\right|\left|\gamma_{T}^{\prime} \Delta^{2} \mathbf{D} \mathbf{T}_{t}\left(\tau_{0}\right)\right| \\
& \leq 4 T^{-1} \sup _{t=1, \ldots, T}\left|u_{t}\right| \sum_{t=3}^{T}\left|\gamma_{T}^{\prime} \Delta^{2} \mathbf{D} \mathbf{T}_{t}\left(\tau_{0}\right)\right| \\
& =4 T^{-1} \sup _{t=1, \ldots, T}\left|u_{t}\right|\left(\iota_{m}^{\prime}\left|\gamma_{T}\right|\right)=O_{p}\left(T^{-1}\right) .
\end{aligned}
$$

Hence, for $\tilde{V}_{T}(r):=T^{-1} \sum_{t=3}^{\lfloor r T\rfloor}\left(\Delta^{2} u_{t}\right)^{2}$ we have that $\sup _{r \in[0,1]}\left|V_{T}(r)-\tilde{V}_{T}(r)\right| \rightarrow{ }_{p} 0$. Therefore, to prove that (A.7) holds we need to show that $\tilde{V}_{T}(r)$ converges to the right member of (A.7). Since $\Delta u_{t}=(-c / T) u_{t-1}+\varepsilon_{t}$, we have that

$$
\begin{aligned}
\Delta^{2} u_{t} & =(-c / T) \Delta u_{t-1}+\Delta \varepsilon_{t} \\
& =(-c / T)^{2} u_{t-2}+(-c / T) \varepsilon_{t-1}+\varepsilon_{t}-\varepsilon_{t-1}
\end{aligned}
$$

which implies, after some simple algebra, and using the facts that $\sup _{t}\left|u_{t}\right|=O_{p}\left(T^{1 / 2}\right)$ and that $\varepsilon_{t}$ has bounded second moments, that

$$
\sup _{r \in[0,1]}\left|\tilde{V}_{T}(r)-T^{-1} \sum_{t=3}^{\lfloor r T\rfloor}\left(\varepsilon_{t}-\varepsilon_{t-1}\right)^{2}\right|=v_{T}
$$


where $v_{T}$ is of $o_{p}(1)$. Since $\varepsilon_{t}-\varepsilon_{t-1}$ has the LP representation

$$
\varepsilon_{t}-\varepsilon_{t-1}=\sum_{i=0}^{\infty} c_{i} e_{t-i}-\sum_{i=1}^{\infty} c_{i-1} e_{t-i}=c_{0} e_{t}+\sum_{i=1}^{\infty}\left(c_{i}-c_{i-1}\right) e_{t-i}=\sum_{i=0}^{\infty} \tilde{c}_{i} e_{t-i}
$$

with $\tilde{c}_{0}:=c_{0}$ and $\tilde{c}_{i}:=\left(c_{i}-c_{i-1}\right), i=1,2, \ldots$, we can proceed as in the proof of Theorem 2 in Cavaliere and Taylor (2007) to show that

$$
\begin{aligned}
T^{-1} \sum_{t=3}^{\lfloor r T\rfloor}\left(\varepsilon_{t}-\varepsilon_{t-1}\right)^{2} \stackrel{p}{\rightarrow} \sum_{i=0}^{\infty} \tilde{c}_{i}^{2}\left(\int_{0}^{r} \omega(u)^{2} d u\right) & =2\left(\sum_{i=0}^{\infty} c_{i}\left(c_{i}-c_{i+1}\right)\right)\left(\int_{0}^{r} \omega(u)^{2} d u\right) \\
& =\kappa^{2}\left(\int_{0}^{r} \omega(s)^{2} d s\right)
\end{aligned}
$$

with $\kappa:=\left(2 \sum_{i=0}^{\infty} c_{i}\left(c_{i}-c_{i+1}\right)\right)^{1 / 2}$. This implies that $\tilde{V}_{T}(r)$, and hence $V_{T}(r)$, both converge in probability to $\kappa^{2} \int_{0}^{r} \omega(s)^{2} d s$, as required. Hence (A.7) and therefore (A.8) hold.

By the bootstrap invariance principle (A.8) and the CMT we then find that

$$
\begin{aligned}
& T^{-3 / 2} \sum_{t=\lfloor r T\rfloor}^{T} y_{t-1}^{*} \stackrel{w}{\rightarrow} p \bar{\omega}^{*} \int_{r}^{1} W_{0}^{\eta}(s) d s \\
& T^{-3 / 2} \sum_{t=\lfloor r T\rfloor}^{T} t \Delta y_{t}^{*} \stackrel{w}{\rightarrow} p \bar{\omega}^{*}\left\{W_{0}^{\eta}(1)-r W_{0}^{\eta}(r)-\int_{r}^{1} W_{0}^{\eta}(s) d s\right\} \\
& T^{-5 / 2} \sum_{t=\lfloor r T\rfloor}^{T} t y_{t-1}^{*} \stackrel{w}{\rightarrow} p \bar{\omega}^{*} \int_{r}^{1} s W_{0}^{\eta}(s) d s .
\end{aligned}
$$

analogously to (A.2)-(A.4) in the proof of Theorem 1. Further, paralleling the arguments in the proof of Theorem 1, and using the fact that $T^{-1 / 2} y_{\lfloor r T\rfloor}^{*}$ does not depend on $c$ or $\boldsymbol{\kappa}$ in the limit,

$$
\left[\begin{array}{c}
\tilde{\mu}^{*} \\
T^{1 / 2} \tilde{\beta}^{*} \\
T^{1 / 2} \tilde{\boldsymbol{\gamma}}^{*}
\end{array}\right] \stackrel{w}{\rightarrow} p\left[\begin{array}{ccc}
1 & 0 & \mathbf{0}^{\prime} \\
0 & a_{\bar{c}} & \mathbf{m}_{\bar{c}}(\boldsymbol{\tau})^{\prime} \\
\mathbf{0} & \mathbf{m}_{\bar{c}}(\boldsymbol{\tau}) & \mathbf{D}_{\bar{c}}(\boldsymbol{\tau})
\end{array}\right]^{-1}\left[\begin{array}{c}
0 \\
\bar{\omega}^{*} b_{0, \bar{c}, \eta} \\
\bar{\omega}^{*} \mathbf{b}_{0, \bar{c}, \eta}(\boldsymbol{\tau})
\end{array}\right]
$$

giving the limit of $T^{-1 / 2} \tilde{u}_{\lfloor r T\rfloor}^{*}$ as

$$
\begin{aligned}
T^{-1 / 2} \tilde{u}_{\lfloor r T\rfloor}^{*}= & T^{-1 / 2} y_{\lfloor r T\rfloor}^{*}-T^{-1 / 2} \tilde{\mu}^{*}-T^{-1 / 2} \tilde{\beta}^{*}\lfloor r T\rfloor-T^{-1 / 2} \tilde{\boldsymbol{\gamma}}^{* \prime}\left\{(\lfloor r T\rfloor-\lfloor\boldsymbol{\tau} T\rfloor) \circ \mathbf{I}_{\boldsymbol{\tau}}^{r}\right\} \\
= & T^{-1 / 2} y_{\lfloor r T\rfloor}^{*}-\left[\begin{array}{c}
r \\
(r-\boldsymbol{\tau}) \circ \mathbf{I}_{\boldsymbol{\tau}}^{r}
\end{array}\right]^{\prime}\left[\begin{array}{c}
T^{1 / 2} \tilde{\beta}^{*} \\
T^{1 / 2} \tilde{\boldsymbol{\gamma}}^{*}
\end{array}\right]+o_{p}(1) \\
& \stackrel{w}{\rightarrow} p \bar{\omega} W_{0}^{\eta}(r)-\left[\begin{array}{c}
r \\
(r-\boldsymbol{\tau}) \circ \mathbf{I}_{\boldsymbol{\tau}}^{r}
\end{array}\right]^{\prime}\left[\begin{array}{cc}
a_{\bar{c}} & \mathbf{m}_{\bar{c}}(\boldsymbol{\tau})^{\prime} \\
\mathbf{m}_{\bar{c}}(\boldsymbol{\tau}) & \mathbf{D}_{\bar{c}}(\boldsymbol{\tau})
\end{array}\right]^{-1}\left[\begin{array}{c}
\bar{\omega}^{*} b_{0, \bar{c}, \eta} \\
\bar{\omega}^{*} \mathbf{b}_{0, \bar{c}, \eta}(\boldsymbol{\tau})
\end{array}\right] \\
= & \bar{\omega}^{*} L_{0, \bar{c}}\left(r, \boldsymbol{\tau}_{0}, \boldsymbol{\tau}, \mathbf{0}, \eta\right) .
\end{aligned}
$$

We now consider the limit behaviour of $\hat{\sigma}_{e}^{* 2}:=(T-2)^{-1} \sum_{t=2}^{T} \hat{e}_{t}^{* 2}$ and show that for any $\epsilon>0$ and conditionally on the original sample,

$$
P^{*}\left(\left|\hat{\sigma}_{e}^{* 2}-\bar{\omega}^{* 2}\right|>\epsilon\right) \stackrel{p}{\rightarrow} 0
$$

Using the weak convergence (in probability) result for $T^{-1 / 2} \tilde{u}_{\lfloor r T\rfloor}^{*}$ and for the bootstrap estimators of the deterministic components in (A.9), it is straightforward to see that, for any $\epsilon>0$, we have that 
$P^{*}\left(\left|\hat{\sigma}_{e}^{* 2}-(T-2)^{-1} \sum_{t=2}^{T} \varepsilon_{t}^{* 2}\right|>\epsilon\right) \rightarrow 0$ in probability. Hence, to prove (A.10) it suffices to show that

$$
P^{*}\left(\left|\frac{1}{T-2} \sum_{t=2}^{T} \varepsilon_{t}^{* 2}-\bar{\omega}^{* 2}\right|>\epsilon\right) \stackrel{p}{\rightarrow} 0,
$$

see also Cavaliere and Taylor (2008, proof of Theorem 2).

To prove (A.11) consider the equalities:

$$
\frac{1}{T} \sum_{t=2}^{T} \varepsilon_{t}^{* 2}=\frac{1}{T} \sum_{t=2}^{T}\left(\Delta^{2} y_{t}\right)^{2} w_{t}^{2}=\frac{1}{T} \sum_{t=2}^{T}\left(\Delta^{2} y_{t}\right)^{2}+\frac{1}{T} \sum_{t=2}^{T}\left(\Delta^{2} y_{t}\right)^{2} \xi_{t}=V_{T}(1)+f_{T}
$$

with $\xi_{t}:=w_{t}^{2}-1$ an independent sequence of centered $\chi^{2}(1)$ random variables, and $f_{T}:=T^{-1} \sum_{t=2}^{T}\left(\Delta^{2} y_{t}\right)^{2} \xi_{t}$. As shown above, see (A.7), we have that $V_{T}(1) \rightarrow \kappa^{2} \int_{0}^{r} \omega(s)^{2} d s=\bar{\omega}^{* 2}$, in probability. Moreover, conditionally on the sample,

$$
\begin{aligned}
E^{*}\left(f_{T}^{2}\right) & =E^{*}\left(\frac{1}{T} \sum_{t=1}^{T}\left(\Delta^{2} y_{t}\right)^{2} \xi_{t}\right)^{2}=\frac{1}{T^{2}} \sum_{t=1}^{T} \sum_{s=1}^{T}\left(\Delta^{2} y_{t}\right)^{2}\left(\Delta^{2} y_{s}\right)^{2} E\left(\xi_{t} \xi_{s}\right)=\frac{1}{T^{2}} \sum_{t=1}^{T}\left(\Delta^{2} y_{t}\right)^{4} E\left(\xi_{t}^{2}\right) \\
& =\frac{4}{T}\left(\frac{1}{T} \sum_{t=1}^{T}\left(\Delta^{2} y_{t}\right)^{4}\right)=\frac{4}{T}\left(\frac{1}{T} \sum_{t=1}^{T}\left(\Delta^{2} u_{t}\right)^{4}\right)+o_{p}\left(T^{-1}\right) \\
& =\frac{4}{T}\left(\frac{1}{T} \sum_{t=1}^{T}\left(\varepsilon_{t}-\varepsilon_{t-1}\right)^{4}\right)+o_{p}\left(T^{-1}\right)=O_{p}\left(T^{-1}\right)
\end{aligned}
$$

since $T^{-1} \sum_{t=1}^{T}\left(\varepsilon_{t}-\varepsilon_{t-1}\right)^{4}$ is of $O_{p}(1)$ under Assumption $\mathcal{A}_{2}$. Hence, we finally have that, for any $\epsilon>0$, a simple (conditional) version of Tchebychev's inequality yields

$$
P^{*}\left(\left|f_{T}\right|>\epsilon\right) \leq \frac{E^{*}\left(f_{T}^{2}\right)}{\epsilon^{2}} \stackrel{p}{\rightarrow} 0
$$

and hence that $f_{T}=o_{p}^{*}(1)$. This proves (A.11).

Finally, we can write,

$$
\begin{aligned}
D F_{\bar{c}}^{G L S}(\boldsymbol{\tau})^{*}= & \frac{\left(T^{-1 / 2} \tilde{u}_{T}^{*}\right)^{2}-T^{-1} \sum_{t=2}^{T}\left(\Delta \tilde{u}_{t}^{*}\right)^{2}}{2 \sqrt{\hat{\sigma}_{e}^{* 2} T^{-2} \sum_{t=2}^{T} \tilde{u}_{t-1}^{* 2}}}+o_{p}^{*}(1) \\
& \stackrel{{ }^{w}}{\rightarrow} \frac{\bar{\omega}^{* 2} L_{0, \bar{c}}\left(1, \boldsymbol{\tau}_{0}, \boldsymbol{\tau}, \mathbf{0}, \eta\right)^{2}-\bar{\omega}^{* 2}}{2 \sqrt{\bar{\omega}^{* 2} \cdot \bar{\omega}^{* 2} \int_{0}^{1} L_{0, \bar{c}}\left(r, \boldsymbol{\tau}_{0}, \boldsymbol{\tau}, \mathbf{0}, \eta\right)^{2} d r}}=\mathcal{D}_{0, \bar{c}}\left(\boldsymbol{\tau}_{0}, \boldsymbol{\tau}, \mathbf{0}, \eta\right) .
\end{aligned}
$$

As in the proof of Theorem 1, the stated result for $M D F_{m}^{*}$ then follows from this fixed $\boldsymbol{\tau}$ representation, using the relevant arguments proved in Zivot and Andrews (1992) and an application of the CMT.

\section{References}

Andrews, D.W.K., and Buchinsky, M. (2001). Evaluation of a three-step method for choosing the number of bootstrap repetitions. Journal of Econometrics 103, 345-386. 
Banerjee, A., Lumsdaine, R. and Stock, J. (1992). Recursive and sequential tests of the unit root and trend break hypotheses: theory and international evidence. Journal of Business and Economics Statistics 10, 271-288.

Carrion-i-Silvestre, J.L., Kim, D. and Perron, P. (2009). GLS-based unit root tests with multiple structural breaks both under the null and the alternative hypotheses. Econometric Theory 25, $1754-1792$.

Cavaliere, G., Harvey, D.I., Leybourne, S.J. and Taylor, A.M.R. (2011). Testing for unit roots in the presence of a possible break in trend and non-stationary volatility. Econometric Theory 27, 957-991.

Cavaliere, G. and Taylor, A.M.R. (2007). Testing for unit roots in time series models with nonstationary volatility. Journal of Econometrics 140, 919-947.

Cavaliere G. and Taylor, A.M.R. (2008). Bootstrap unit root tests for time series with non-stationary volatility. Econometric Theory 24, 43-71.

Cavaliere G. and Taylor, A.M.R. (2009). Bootstrap $M$ unit root tests. Econometric Reviews 28, 393-421.

Davidson J. (1994). Stochastic limit theory. Oxford: Oxford University Press.

Elliott, G., Rothenberg, T.J. and Stock, J.H. (1996). Efficient tests for an autoregressive unit root. Econometrica 64, 813-836.

Giné, E. and Zinn, J. (1990). Bootstrapping general empirical measures. Annals of Probability 18, 851-869.

Hansen, B.E. (1996). Inference when a nuisance parameter is not identified under the null hypothesis. Econometrica 64, 413-430.

Harris, D., Harvey, D.I., Leybourne, S.J. and Taylor, A.M.R. (2009). Testing for a unit root in the presence of a possible break in trend. Econometric Theory 25, 1545-1588.

Harvey, D.I., Leybourne, S.J. and Taylor, A.M.R. (2012). Unit root testing under a local break in trend. Journal of Econometrics 167, 140-167.

Harvey, D.I., Leybourne, S.J. and Taylor, A.M.R. (2013). Testing for unit roots in the possible presence of multiple trend breaks using minimum Dickey-Fuller statistics. Journal of Econometrics $177,265-284$.

Im, K.S., Pesaran, M.H. and Shin, Y. (2003). Testing for unit roots in heterogeneous panels. Journal of Econometrics 115, 53-74. 
Kim, D. and Perron, P. (2009). Unit root tests allowing for a break in the trend function at an unknown time under both the null and alternative hypotheses. Journal of Econometrics 148, $1-13$.

Liu, R.Y. (1988). Bootstrap procedures under some non i.i.d. models. Annals of Statistics 16, 1696-1708.

Mammen, E. (1993). Bootstrap and wild bootstrap for high dimensional linear models. Annals of Statistics 21, 255-285.

Perron, P. (1989). The great crash, the oil price shock, and the unit root hypothesis. Econometrica $57,1361-1401$.

Perron, P. (1997). Further evidence of breaking trend functions in macroeconomic variables. Journal of Econometrics 80, 355-385.

Perron, P. and Rodríguez, G. (2003). GLS detrending, efficient unit root tests and structural change. Journal of Econometrics 115, 1-27.

Vogelsang, T.J. and Perron, P. (1998). Additional tests for a unit root allowing the possibility of breaks in the trend function. International Economic Review 39, 1073-1100.

Zivot, E. and Andrews, D.W.K. (1992). Further evidence on the great crash, the oil-price shock, and the unit-root hypothesis. Journal of Business and Economic Statistics 10, 251-270. 


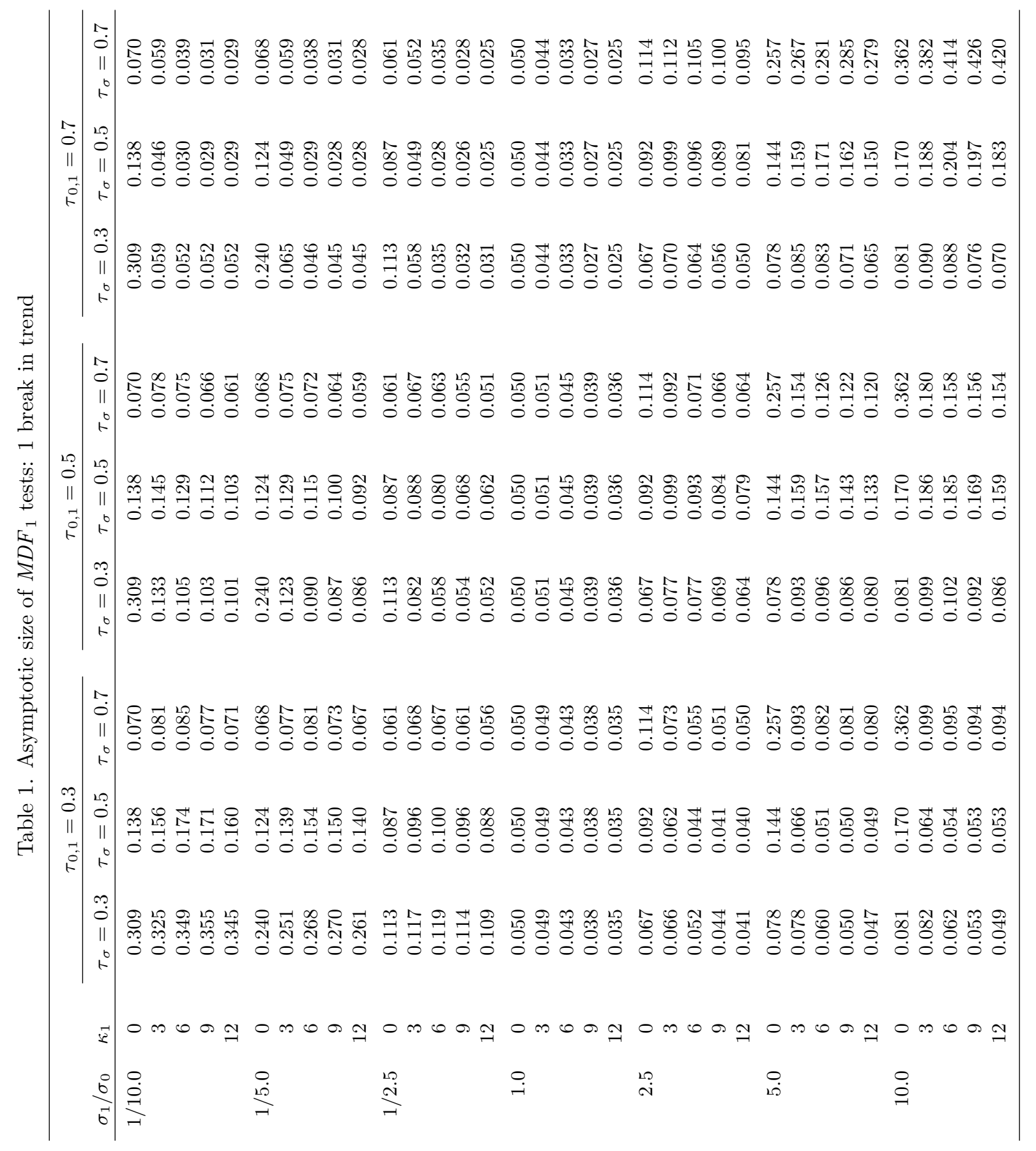




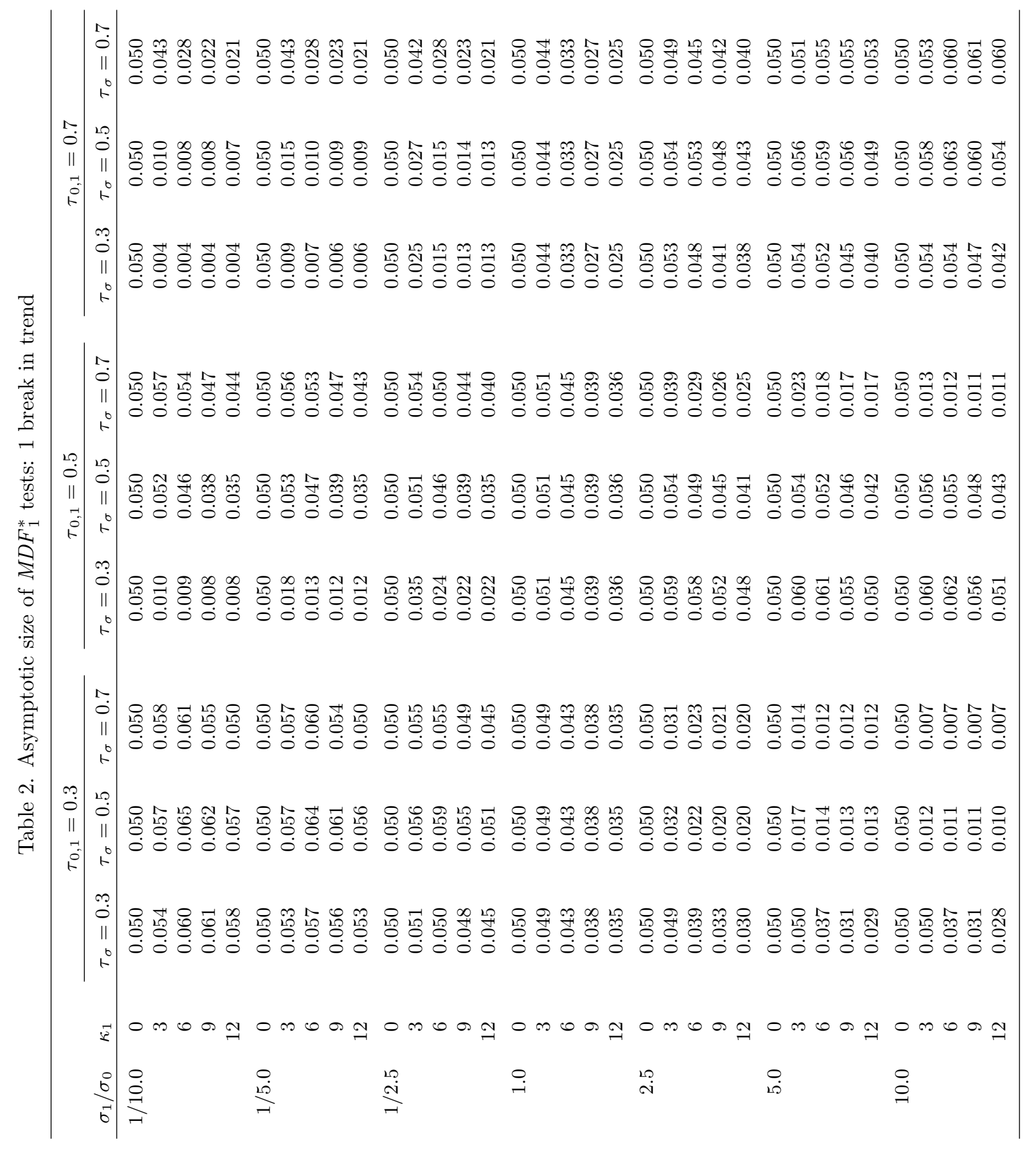




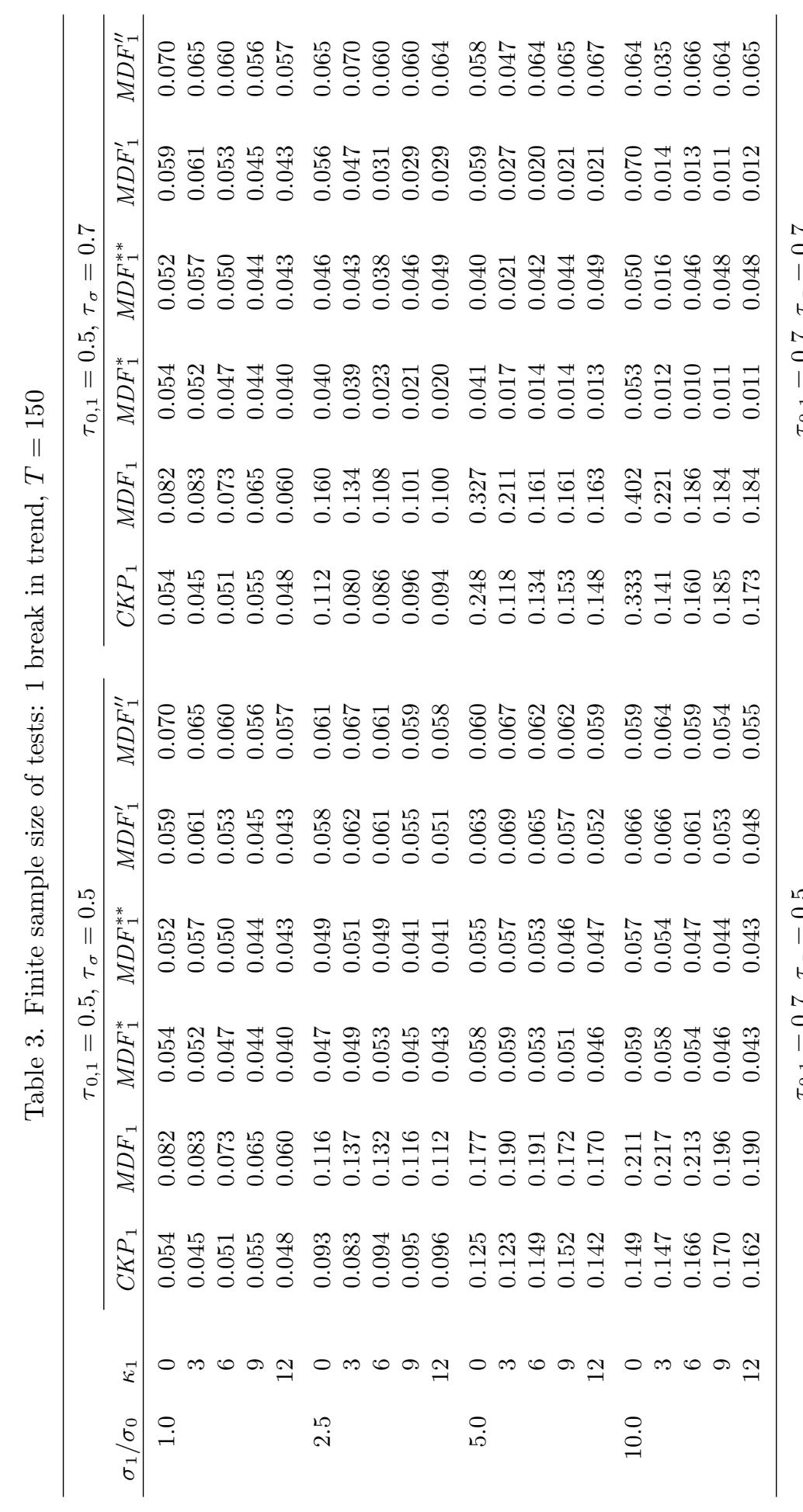

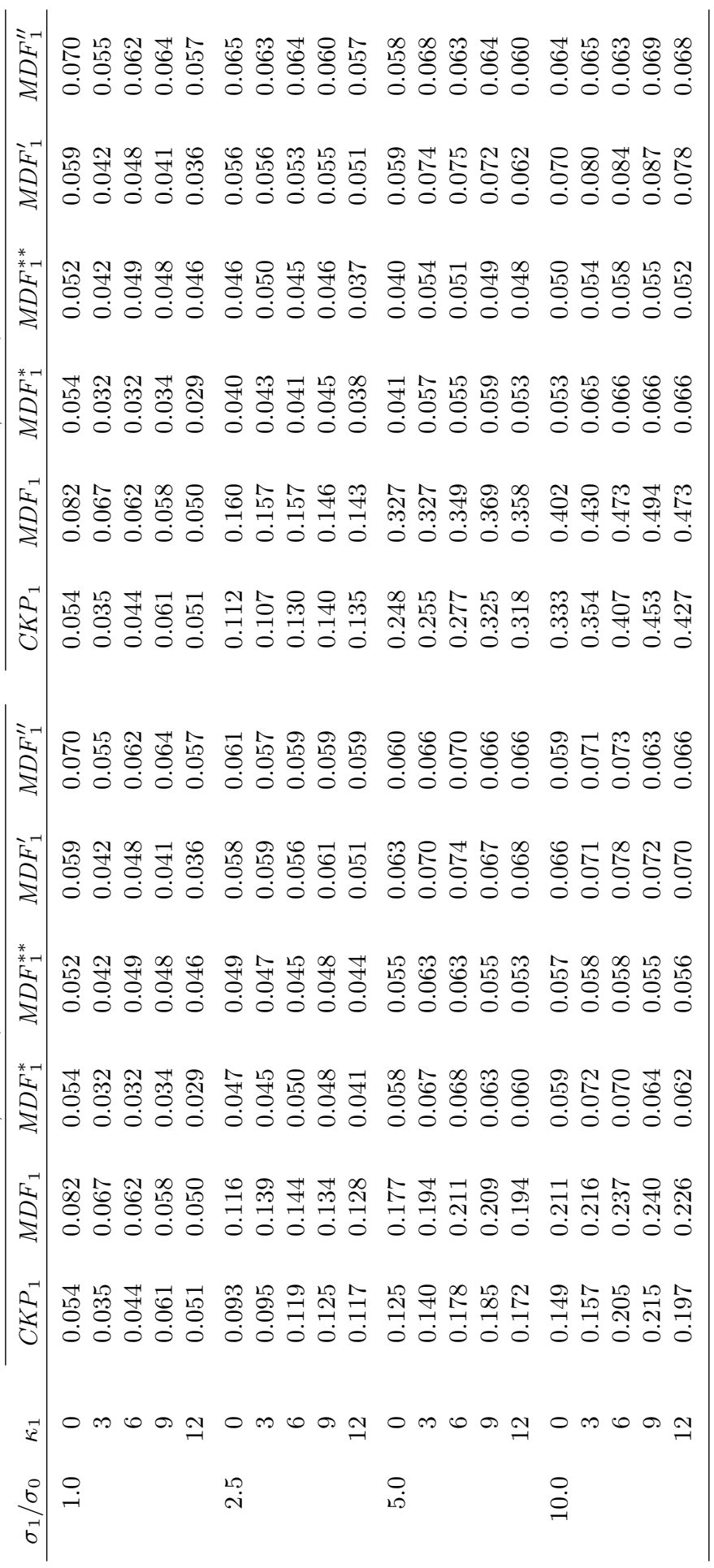




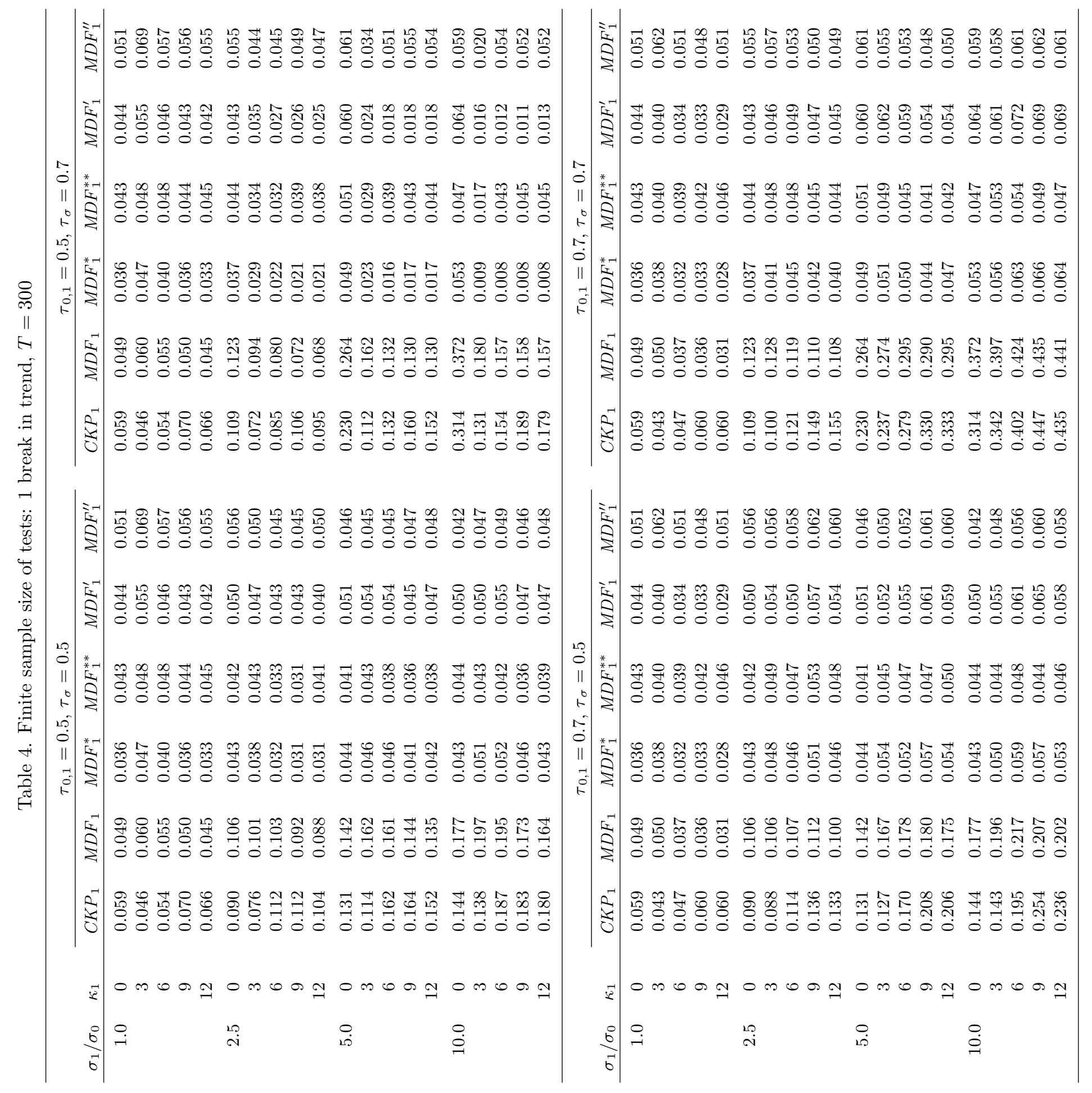




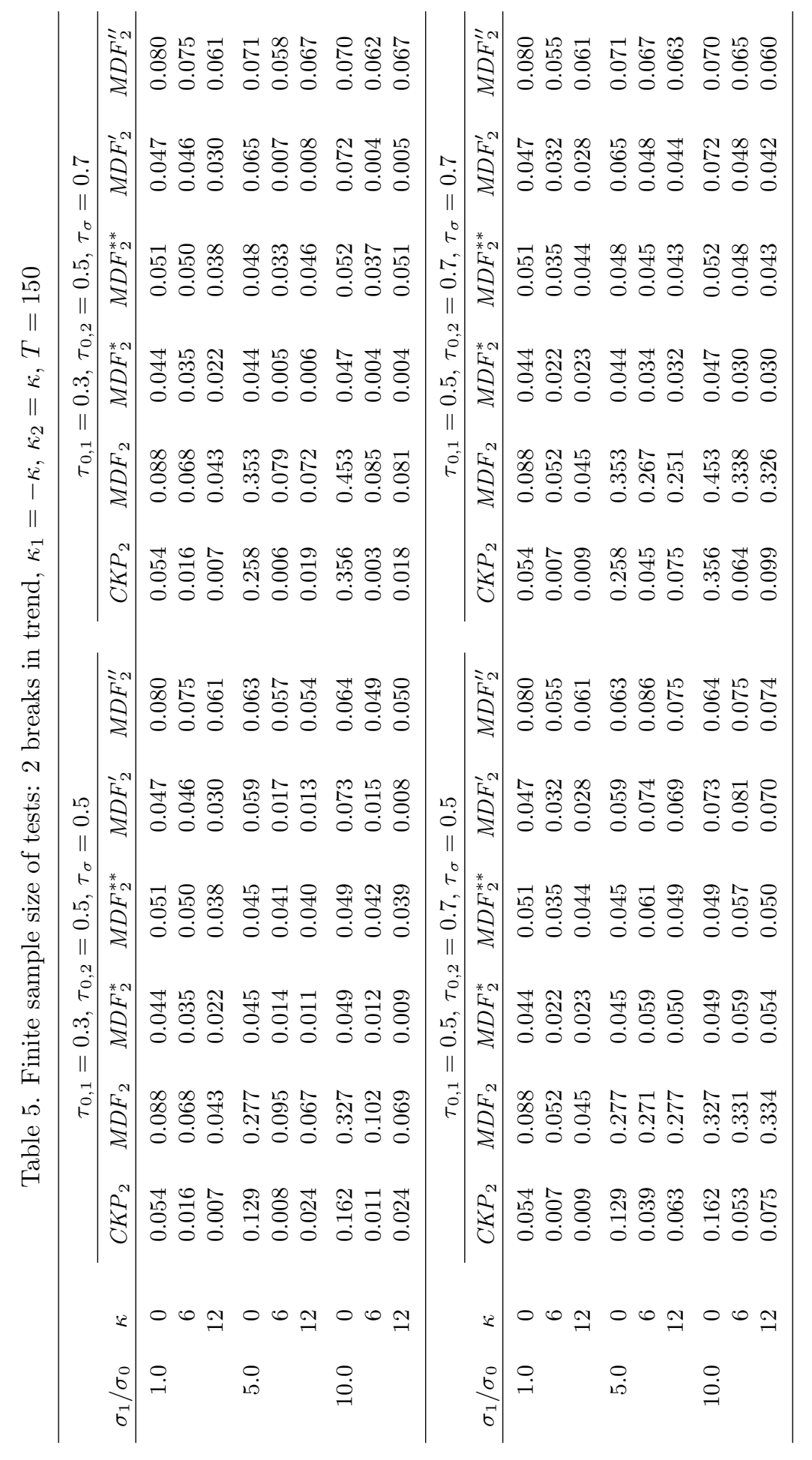




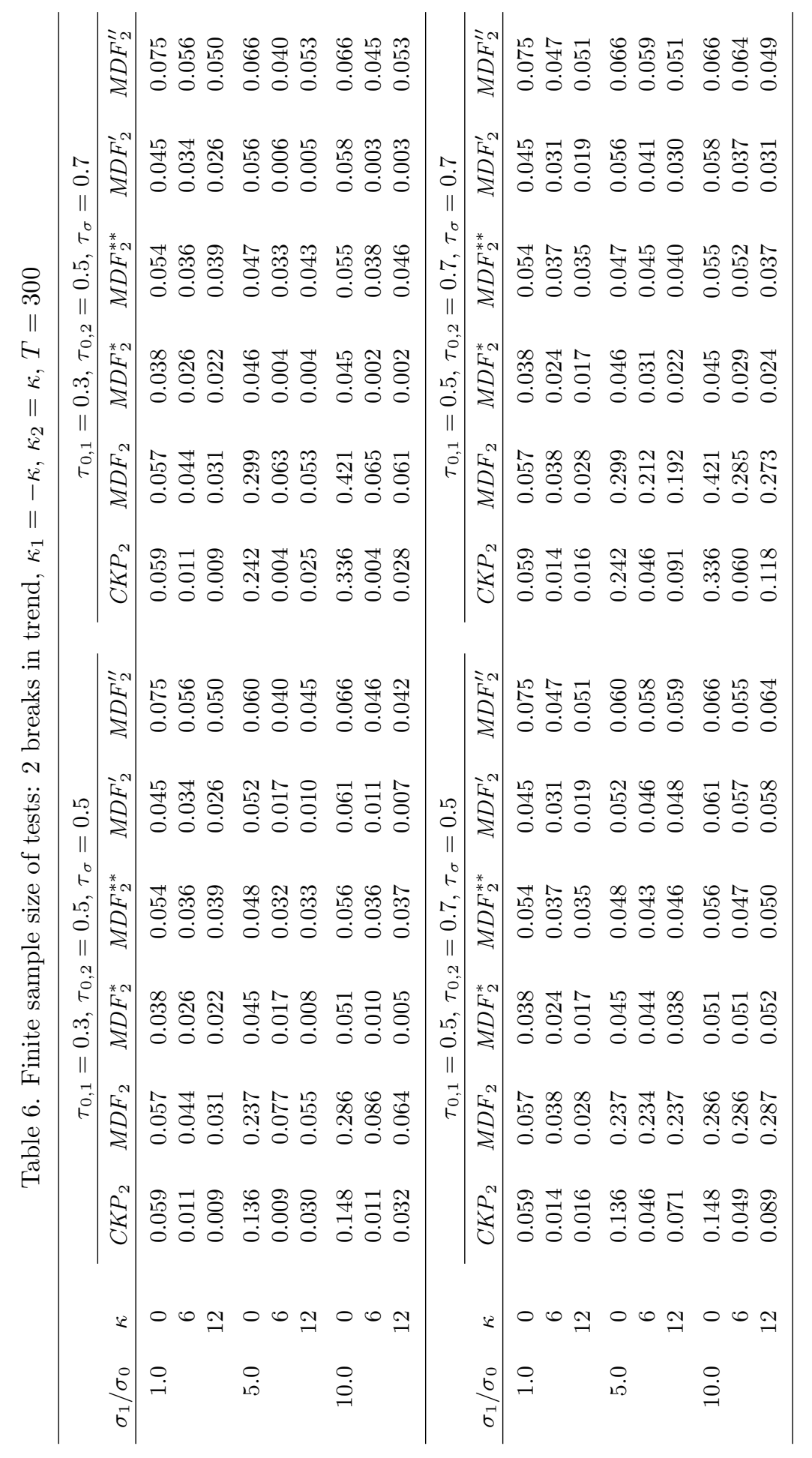




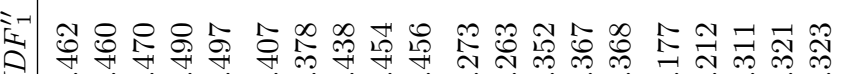

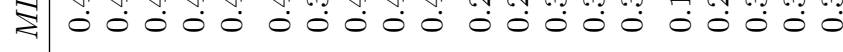

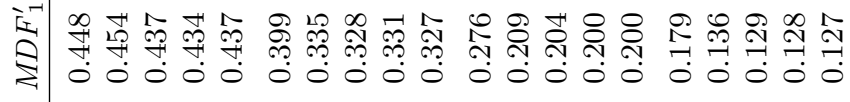

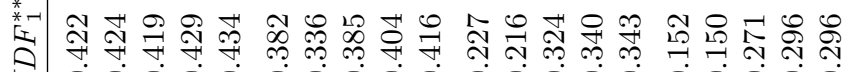

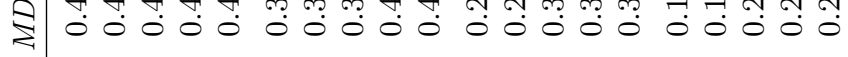

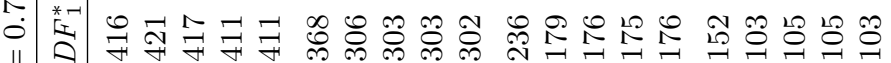

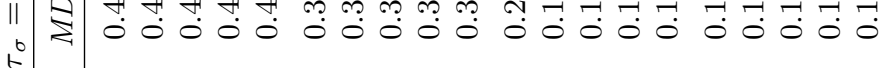
เి. छ

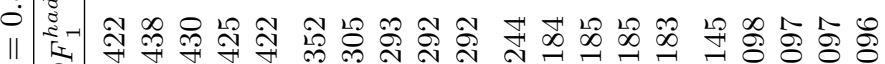

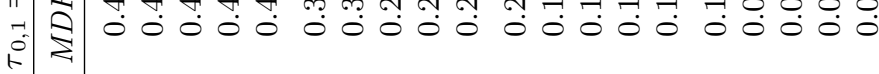

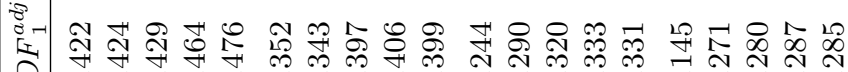
O.

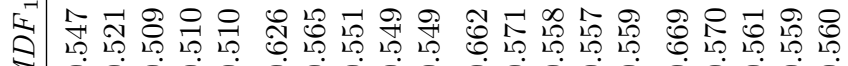

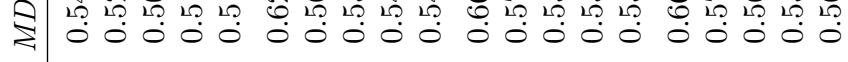

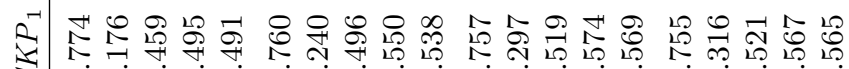

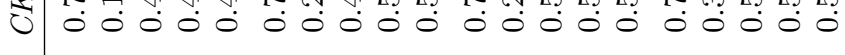

侙

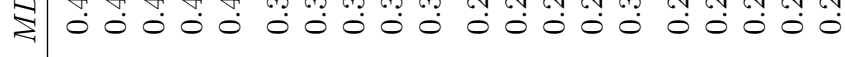

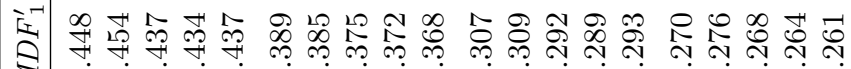

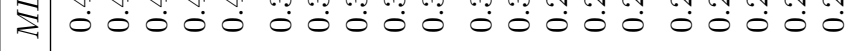

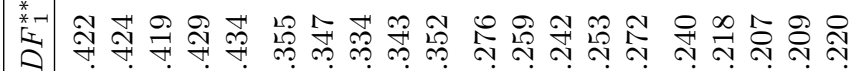

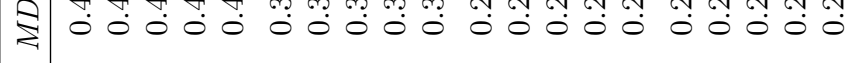

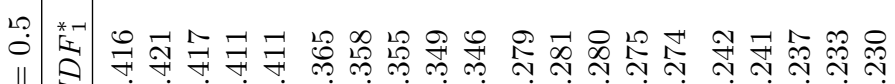
॥. نุ.

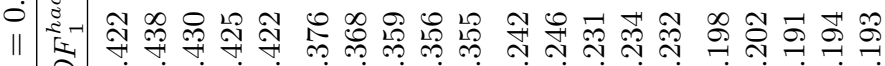

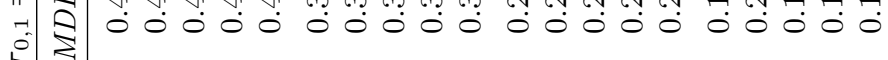

荧 娄

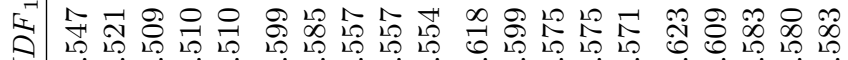

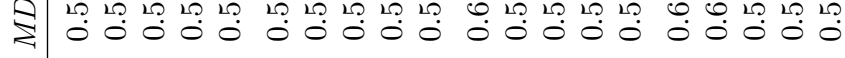

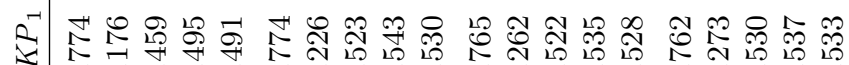

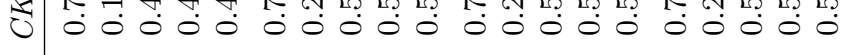

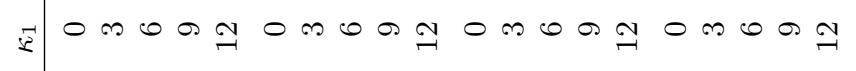$$
\stackrel{\circ}{b} \mid \stackrel{0}{6}
$$$$
\text { เ }
$$

$\stackrel{4}{10}$

$\underset{20}{0}$

$\stackrel{\circ}{\circ}$

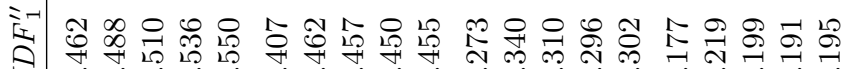

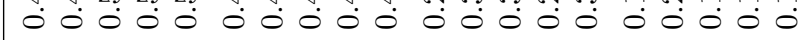

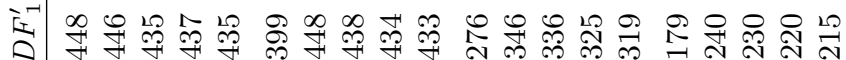
00000000000000000000

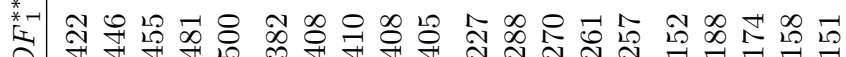

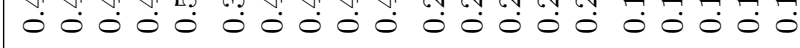

㳦

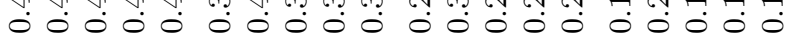

|| -

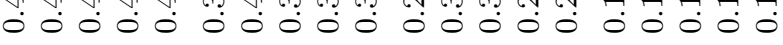

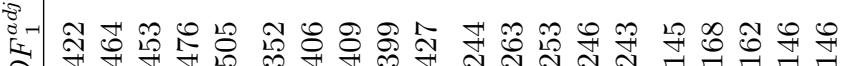

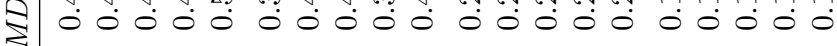

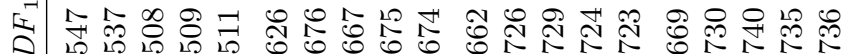

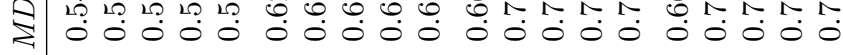

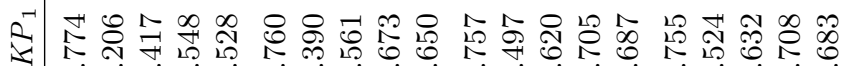

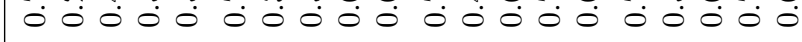

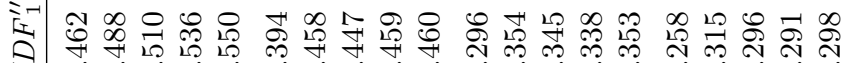

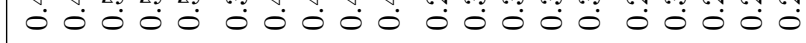

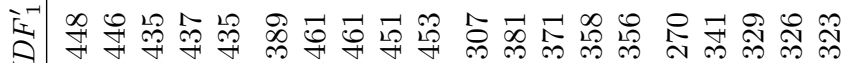

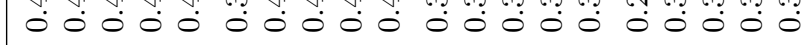

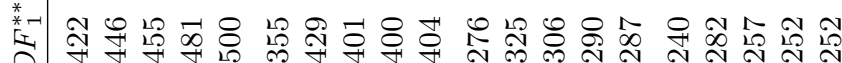

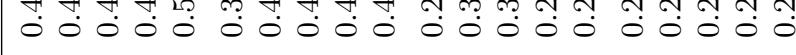

承

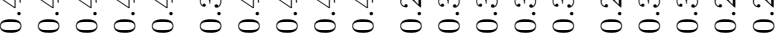

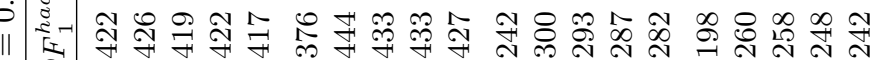

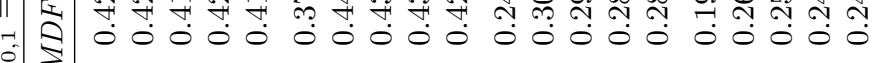

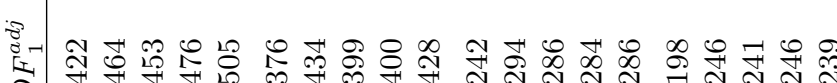
$\exists$ :

넌

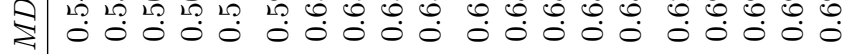

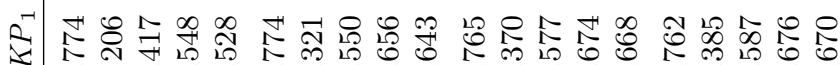

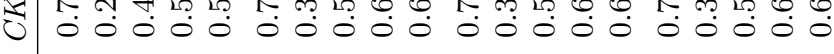

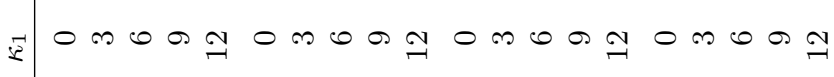

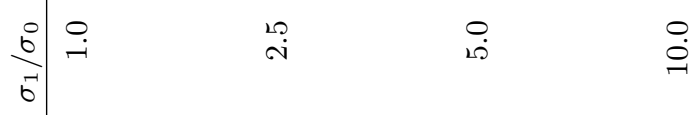


过

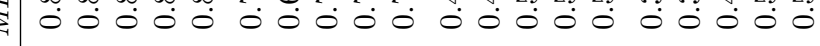

氙

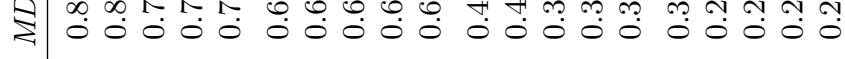

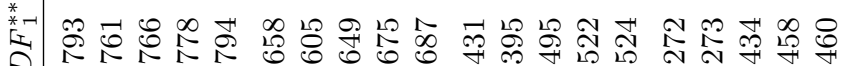

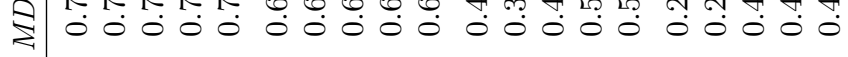
닌

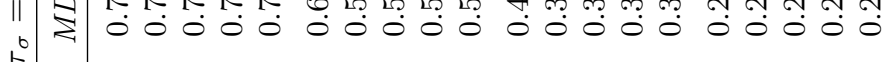
ن.

||

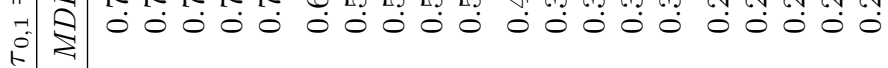

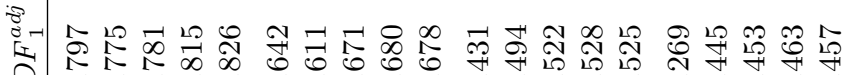

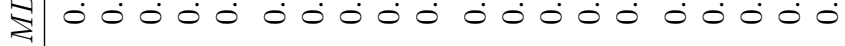

近

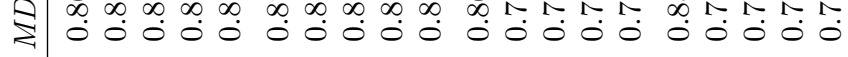

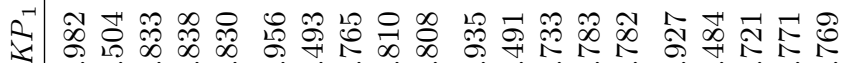

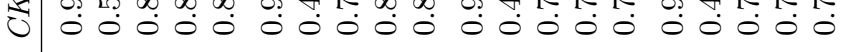

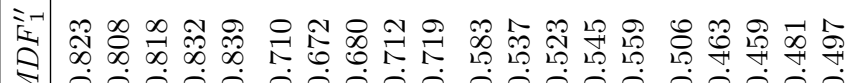

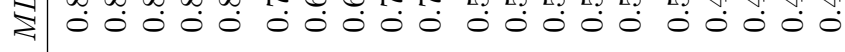

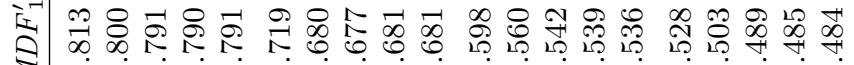

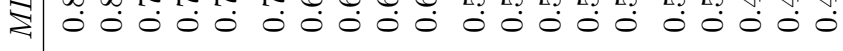

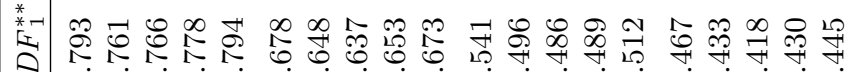

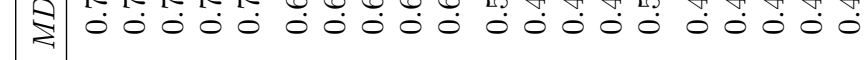

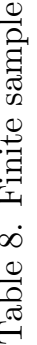

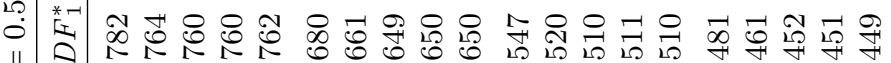

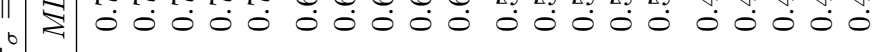
:

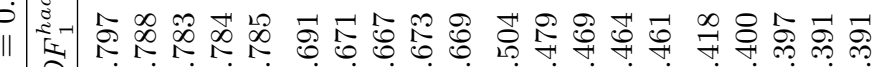

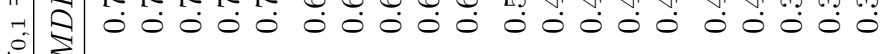

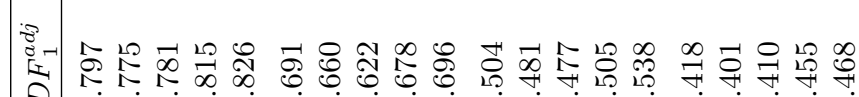

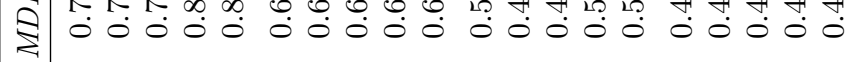

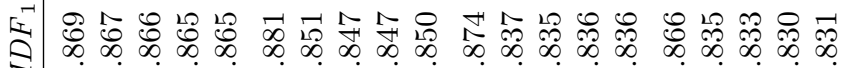

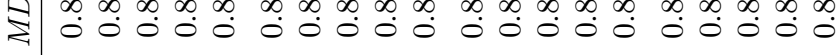

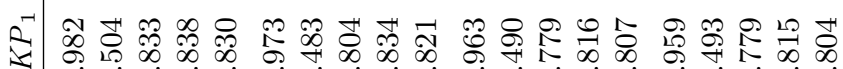

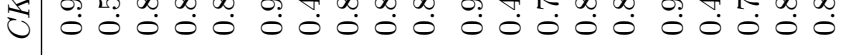

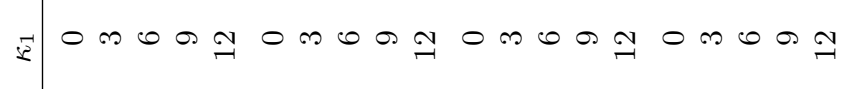
$\frac{0}{6}$ ก.

$\stackrel{10}{i}$

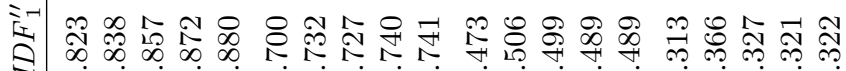

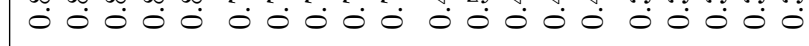

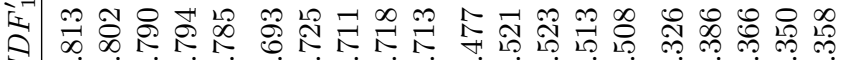

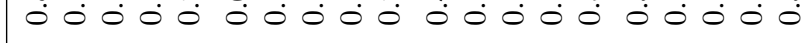

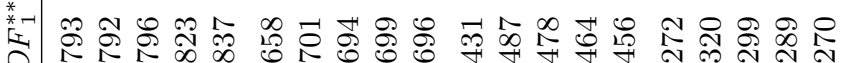

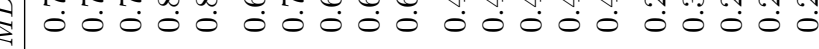

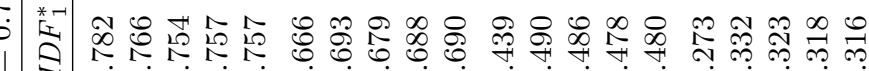
0000000

|

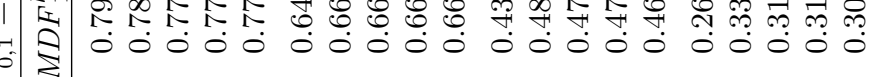

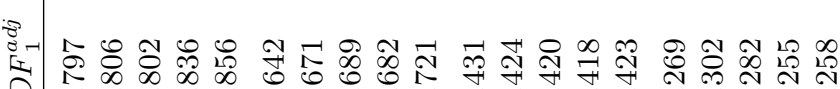

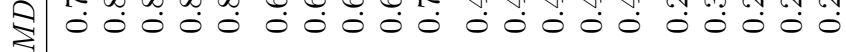

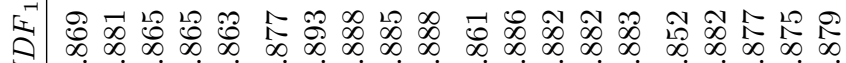

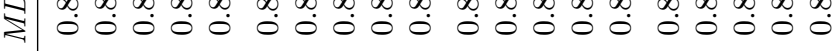

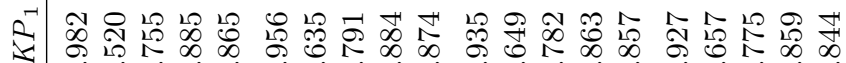

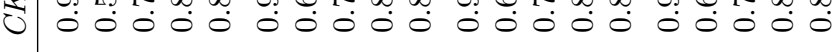

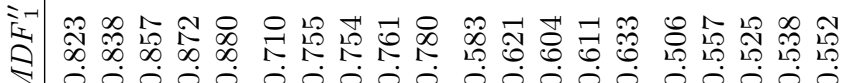
\begin{tabular}{llllllllllllllllllllll}
\hline & 0 & 0 & 0 & 0 & 0 & 0 & 0 & 0 & 0 & 0 & 0 & 0 & 0 & 0 & 0 & 0 & 0 & 0 & 0
\end{tabular}

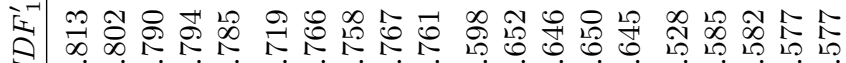

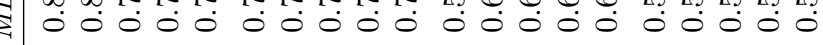

*

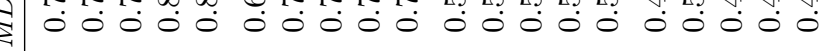

*

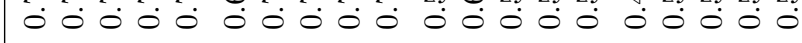

||

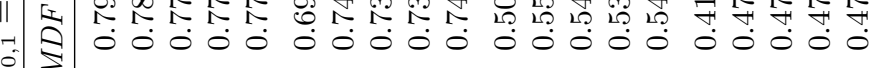

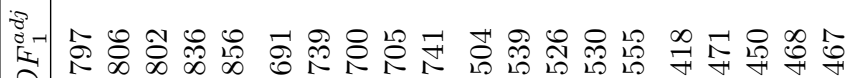

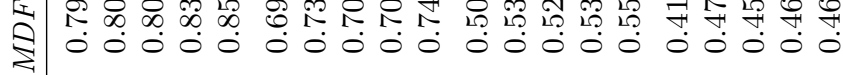

I

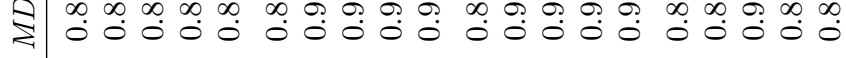

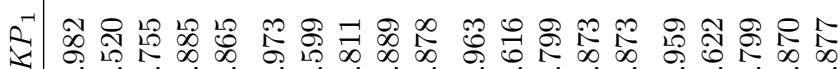

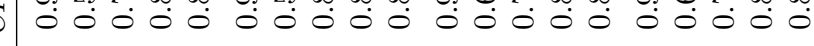
T) \begin{tabular}{c|cccc}
0 & 0 & 0 & 0 & 0 \\
0 & -1 & 4 & 10 & 0 \\
& & & & 0
\end{tabular} 


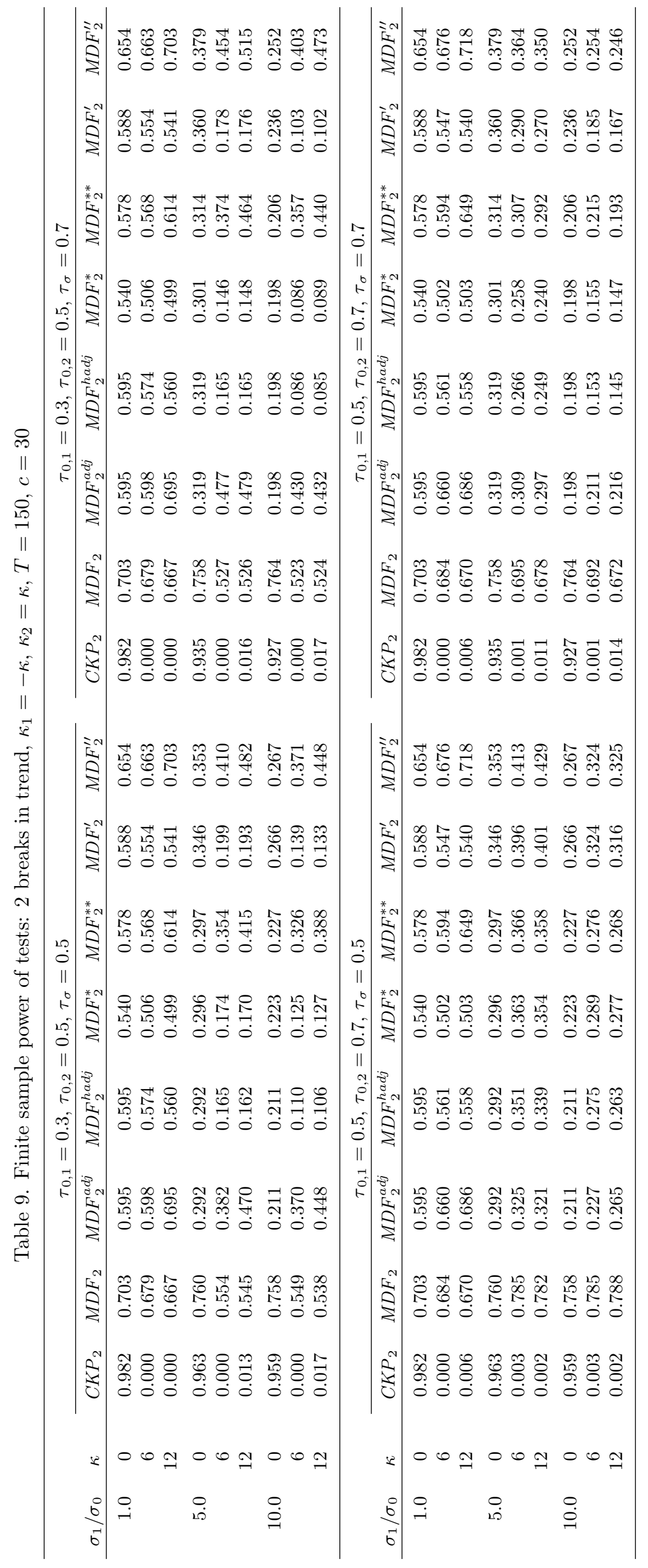

